\title{
Hodge decomposition of string topology
}

\author{
Yuri Berest ${ }^{1}$, Ajay C. Ramadoss ${ }^{2}$ and Yining Zhang ${ }^{3}$ \\ ${ }^{1}$ Department of Mathematics, Cornell University, Ithaca, NY 14853-4201, USA; E-mail: berest@math.cornell.edu. \\ ${ }^{2}$ Department of Mathematics, Indiana University, Bloomington, IN 47405, USA; E-mail: ajcramad@indiana.edu. \\ ${ }^{3}$ Department of Mathematics, University of Colorado Boulder, Boulder, CO 80309, USA; E-mail: yining.zhang@colorado.edu.
}

Received: 29 February 2020; Revised: 15 February 2021; Accepted: 5 March 2021

2020 Mathematics Subject Classification: Primary - 55P50; Secondary - 19D55

\begin{abstract}
Let $X$ be a simply connected closed oriented manifold of rationally elliptic homotopy type. We prove that the string topology bracket on the $S^{1}$-equivariant homology $\overline{\mathrm{H}}_{*}^{S^{1}}(\mathcal{L} X, \mathbb{Q})$ of the free loop space of $X$ preserves the Hodge decomposition of $\overline{\mathrm{H}}_{*}^{S^{1}}(\mathcal{L} X, \mathbb{Q})$, making it a bigraded Lie algebra. We deduce this result from a general theorem on derived Poisson structures on the universal enveloping algebras of homologically nilpotent finite-dimensional DG Lie algebras. Our theorem settles a conjecture of [7].
\end{abstract}

\section{Introduction}

Let $X$ be a simply connected closed oriented manifold, and let $\mathcal{L} X:=\operatorname{Map}\left(S^{1}, X\right)$ denote the free loop space over $X$. Chas and Sullivan [14] showed that the rational equivariant homology of $\mathcal{L} X$ with respect to the natural $S^{1}$-action carries a graded Lie algebra structure with the so-called string topology bracket

$$
\{-,-\}: \overline{\mathrm{H}}_{*}^{S^{1}}(\mathcal{L} X, \mathbb{Q}) \times \overline{\mathrm{H}}_{*}^{S^{1}}(\mathcal{L} X, \mathbb{Q}) \rightarrow \overline{\mathrm{H}}_{*}^{S^{1}}(\mathcal{L} X, \mathbb{Q}) .
$$

This bracket is intrinsically related to the geometry of $\mathcal{L} X$ and has many interesting properties which have been studied extensively in recent years (see, e.g., [24, 25, 56, 57]).

In this paper we show that the string topology bracket (1.1) is compatible with Frobenius (power) operations on $\overline{\mathrm{H}}_{*}^{S^{1}}(\mathcal{L} X, \mathbb{Q})$ - that is, it respects the natural decomposition

$$
\overline{\mathrm{H}}_{*}^{S^{1}}(\mathcal{L} X, \mathbb{Q})=\bigoplus_{p=0}^{\infty} \overline{\mathrm{H}}_{*}^{S^{1},(p)}(\mathcal{L} X, \mathbb{Q}),
$$

where the direct summands are common eigenspaces of graded endomorphisms of $\overline{\mathrm{H}}_{*}^{S^{1}}(\mathcal{L} X, \mathbb{Q})$ with eigenvalues $n^{p}(n \geq 0)$ induced by the finite coverings of the circle $S^{1} \rightarrow S^{1}, e^{i \theta} \mapsto e^{n i \theta}$ [11]. More precisely, we prove the following:

Theorem 1.1. Assume that the manifold $X$ is rationally elliptic as a topological space - that is, $\operatorname{dim} \sum_{i \geq 2} \pi_{i}(X) \otimes \mathbb{Q}<\infty$. Then

$$
\left\{\overline{\mathrm{H}}_{*}^{S^{1},(p)}(\mathcal{L} X, \mathbb{Q}), \overline{\mathrm{H}}_{*}^{S^{1},(q)}(\mathcal{L} X, \mathbb{Q})\right\} \subseteq \overline{\mathrm{H}}_{*}^{S^{1},(p+q-1)}(\mathcal{L} X, \mathbb{Q}), \quad \forall p, q \geq 0, p+q \geq 1 .
$$

(C) The Author(s), 2021. Published by Cambridge University Press. This is an Open Access article, distributed under the terms of the Creative Commons Attribution licence (http://creativecommons.org/licenses/by/4.0/), which permits unrestricted re-use, distribution, and reproduction in any medium, provided the original work is properly cited 
Thus, the Chas-Sullivan Lie algebra of $X$ is bigraded:

$$
\overline{\mathrm{H}}_{*}^{S^{1}}(\mathcal{L} X, \mathbb{Q})=\bigoplus_{n \geq 0} \bigoplus_{p \geq-1} \overline{\mathrm{H}}_{n}^{S^{1},(p+1)}(\mathcal{L} X, \mathbb{Q}),
$$

where the first grading is given by the homological degree and the second by the Hodge degree (shifted by 1).

The result of this theorem was conjectured in our earlier paper [7], where we showed that the bracket (1.1) preserves a filtration on the vector space $\overline{\mathrm{H}}_{*}^{S^{1}}(\mathcal{L} X, \mathbb{Q})$ associated naturally with the direct sum decomposition (1.2) (see [7, Theorem 4.3]). Thus we strengthen [7, Theorem 4.3], albeit under the additional assumption that $X$ is rationally elliptic. ${ }^{1}$

The question of compatibility of Hodge decomposition with various natural operations, including string topology operations, has been studied by numerous authors (see, e.g., [24, 28, 29, 33, 60] and references therein). In particular, we should mention that [7, Theorem 4.3] can be deduced from results of Felix and Thomas [24]. Specifically, [24, Theorem 2] says that the so-called loop product on the ordinary homology $\overline{\mathrm{H}}_{*}(\mathcal{L} X ; \mathbb{Q})$ preserves its Hodge filtration. Since the Chas-Sullivan bracket $(1.1)$ is determined by the loop product (see $[14,25]$ and equation (4.11)) and the Frobenius operations on $\overline{\mathrm{H}}_{*}(\mathcal{L} X ; \mathbb{Q})$ are compatible with those on $\overline{\mathrm{H}}_{*}^{S^{1}}(\mathcal{L} X, \mathbb{Q})$, one can check that [24, Theorem 2] implies [7, Theorem 4.3]. By contrast - to the best of our knowledge - the result of Theorem 1.1 was not anticipated in the earlier literature.

Theorem 1.1 is a geometric fact: it relates two geometrically defined structures on a simply connected manifold $X$. Unfortunately, we do not know how to see this relation directly, in geometric terms, using the original definition of string topology in [14]. Instead, we prove Theorem 1.1 in a somewhat roundabout way, deducing it from an abstract algebraic result on derived Poisson structures on the universal enveloping algebra $\mathcal{U} \mathfrak{a}$ of a (DG) Lie algebra $\mathfrak{a}$ (see [2]). The main property of such a structure is that it naturally induces a Lie bracket on the (reduced) cyclic homology of $\mathcal{U} \mathfrak{a}$ :

$$
\{-,-\}: \overline{\mathrm{HC}}_{*}(\mathcal{U a}) \times \overline{\mathrm{HC}}_{*}(\mathcal{U a}) \rightarrow \overline{\mathrm{HC}}_{*}(\mathcal{U a}),
$$

which is an algebraic model for the string topology bracket (1.1). On the other hand, for any DG Lie algebra $\mathfrak{a}$, the cyclic homology of $\mathcal{U} \mathfrak{a}$ has a canonical direct sum decomposition

$$
\overline{\mathrm{HC}}_{*}(\mathcal{U} \mathfrak{a})=\bigoplus_{p=1}^{\infty} \mathrm{HC}_{*}^{(p)}(\mathfrak{a}),
$$

which is called the Lie-Hodge decomposition (see [3, 7] and Section 2.2). This raises a natural question about compatibility of the two structures:

Does the derived Poisson bracket (1.3) preserve the Lie-Hodge decomposition (1.4)?

As shown in [7], the answer to this question is, in general, negative. It is therefore necessary to impose certain restrictions on the Lie algebra $\mathfrak{a}$ and the derived Poisson structure on $\mathcal{U} \mathfrak{a}$. In this paper, we consider a special class of derived Poisson structures on $\mathcal{U} \mathfrak{a}$ that arise from a cyclic pairing on a cocommutative DG coalgebra $C$ Koszul dual to the DG Lie algebra $\mathfrak{a}$. Then, under natural finiteness assumptions on $\mathfrak{a}$ and $C$, we prove that the bracket (1.3) does preserve the Lie-Hodge decomposition (1.4). To state our main result in precise terms, we recall that every DG Lie algebra $\mathfrak{a}$ has a minimal model, which is given by an

\footnotetext{
${ }^{1}$ Recall that in rational homotopy theory, there is a fundamental dichotomy dividing all simply connected spaces with finite rational homology into two classes: elliptic and hyperbolic. Although 'generic' spaces are known to be rationally hyperbolic, many important spaces occurring 'in nature' are rationally elliptic: these include, for example, the spheres $S^{n}(n \geq 2)$, the complex projective spaces $\mathbb{C P}^{r}(r \geq 1)$, all compact connected Lie groups $G$ and their homogeneous spaces $G / K$ with $K$ compact connected. Moreover, any simply connected compact manifold $X$ of dimension $d$ is known to be rationally elliptic if $\pi_{i}(X) \otimes \mathbb{Q}=0$ for $i>d$ (see [23, Part IV, Section 32]).
} 
$L_{\infty}$-algebra structure on the homology $\mathrm{H}_{*}(\mathfrak{a})$ of $\mathfrak{a}$ together with an $L_{\infty}$-quasi-isomorphism $\mathfrak{a} \stackrel{\sim}{\rightarrow} \mathrm{H}_{*}(\mathfrak{a})$. This structure is unique up to an $L_{\infty}$-quasiautomorphism $\mathrm{H}_{*}(\mathfrak{a}) \rightarrow \mathrm{H}_{*}(\mathfrak{a})$, whose first component is the identity map on $\mathrm{H}_{*}(\mathfrak{a})$ (see [47, Theorem 10.3.15]). We denote this minimal $L_{\infty}$-model simply by $\mathrm{H}_{*}(\mathfrak{a})$.

Theorem 1.2. Let $\mathfrak{a} \in \mathrm{DGLA}_{k}^{+}$be a nonnegatively graded DG Lie algebra defined over a field $k$ of characteristic 0. Assume the following:

1. $\operatorname{dim}_{k} \mathrm{H}_{*}(\mathfrak{a})<\infty$, and $\mathrm{H}_{*}(\mathfrak{a})$ is nilpotent as an $L_{\infty}$-algebra.

2. a has a Koszul-dual cocommutative coalgebra $C$ with $\operatorname{dim}_{k} C<\infty$.

Then the derived Poisson bracket (1.3) associated to a(ny) nondegenerate cyclic pairing on C preserves the Lie-Hodge decomposition (1.4) - that is,

$$
\left\{\mathrm{HC}_{*}^{(p)}(\mathfrak{a}), \mathrm{HC}_{*}^{(q)}(\mathfrak{a})\right\} \subseteq \mathrm{HC}_{*}^{(p+q-2)}(\mathfrak{a}), \quad \forall p, q \geq 0 .
$$

As an example, we note that Theorem 1.2 applies to an ordinary finite-dimensional nilpotent Lie algebra $\mathfrak{a}$, with derived Poisson bracket coming from the natural pairing on the Chevalley-Eilenberg chain complex $\mathcal{C}_{*}(\mathfrak{a} ; k)=\wedge^{*} \mathfrak{a}($ see $[15$, Section 6$])$.

Now, Theorem 1.1 is a consequence of Theorem 1.2 modulo known results in the literature. First of all, we recall that by a fundamental theorem of Quillen [53], the rational homotopy type of any simply connected space $X$ is determined by a DG Lie algebra $\mathfrak{a}_{X}$ called the Quillen model of $X$. To deduce Theorem 1.1 from Theorem 1.2, we thus take $\mathfrak{a}=\mathfrak{a}_{X}$ to be the Quillen model of a given simply connected manifold $X$. Then, by a theorem of Goodwillie [32, Theorem V.1.1] combined with [23, Theorem 26.5], there is a natural isomorphism of graded vector spaces $\overline{\mathrm{HC}}_{*}(\mathcal{U} \mathfrak{a}) \cong \overline{\mathrm{H}}_{*}^{S^{1}}(\mathcal{L} X, \mathbb{Q})$; moreover, by [7, Theorem 1.2], this isomorphism identifies $\operatorname{HC}^{(p)}(\mathfrak{a}) \cong \overline{\mathrm{H}}_{*}^{S^{1},(p-1)}(\mathcal{L} X, \mathbb{Q})$ for all $p \geq 1$. Thus, the geometric Hodge decomposition (1.2) for a simply connected space $X$ coincides (up to a shift in degree) with the Lie-Hodge decomposition (1.4) for the Quillen model of $X$. Next, for any compact manifold $X$, Lambrechts and Stanley [41] constructed a finite-dimensional commutative algebra model $A_{X}$ whose (linear) dual coalgebra $C:=\operatorname{Hom}\left(A_{X}, \mathbb{Q}\right)$ is $\operatorname{Koszul}$ dual to the Quillen model $\mathfrak{a}_{X}$. This coalgebra $C$ comes equipped with a nondegenerate cyclic pairing (Poincaré duality), and - as observed in [2] (see also Lemma 4.3) - the associated derived Poisson bracket on $\overline{\mathrm{HC}}_{*}(\mathcal{U} \mathfrak{a})$ agrees with the ChasSullivan bracket on $\overline{\mathrm{H}}_{*}^{S^{1}}(\mathcal{L} X, \mathbb{Q})$. To apply Theorem 1.2, it remains to note that for $X$ rationally elliptic, the minimal $L_{\infty}$-model of $\mathfrak{a}_{X}$ is finite dimensional and nilpotent - that is, $\mathrm{H}_{*}(\mathfrak{a})$ satisfies condition (1) of Theorem 1.2.

Next, we briefly outline our proof of Theorem 1.2. As a first step, we replace the cyclic homology $\overline{\mathrm{HC}}_{*}(\mathcal{U} \mathfrak{a})$ of the algebra $\mathcal{U}_{\mathfrak{a}}$ by its Hochschild cohomology $\mathrm{HH}^{*}(\mathcal{U} \mathfrak{a}, \mathcal{U} \mathfrak{a})$ and, following an idea of [15], express the derived Poisson bracket on $\overline{\mathrm{HC}}_{*}(\mathcal{U} \mathfrak{a})$ in terms of the canonical cup product on $\mathrm{HH}^{*}\left(\mathcal{U} \mathfrak{a}, \mathcal{U}_{\mathfrak{a}}\right)$ (see Proposition 3.2). We show that the Lie-Hodge decomposition of $\overline{\mathrm{HC}}_{*}(\mathcal{U} \mathfrak{a})$ naturally extends to a direct sum decomposition of $\mathrm{HH}^{*}(\mathcal{U} \mathfrak{a}, \mathcal{U} \mathfrak{a})$, which we also refer to as a Lie-Hodge decomposition (see Theorem 2.2). Using the results of [7, 15], we then reduce the proof of Theorem 1.2 to proving that the cup product on $\mathrm{HH}^{*}\left(\mathcal{U}_{\mathfrak{a}}, \mathcal{U}_{\mathfrak{a}}\right)$ preserves its Lie-Hodge decomposition. Writing $\mathcal{A}$ for the ChevalleyEilenberg cochain complex of the minimal $L_{\infty}$-model $\mathrm{H}_{*}(\mathfrak{a})$ of the DG Lie algebra $\mathfrak{a}$, we observe that there is a Hodge-degree-preserving algebra isomorphism $\operatorname{HH}^{*}\left(\mathcal{U}_{\mathfrak{a}}, \mathcal{U}_{\mathfrak{a}}\right) \cong \operatorname{HH}_{\oplus}^{*}(\mathcal{A}, \mathcal{A})$, where $\mathrm{HH}_{\oplus}^{*}(\mathcal{A}, \mathcal{A})$ stands for the Hochschild cochain complex of $\mathcal{A}$ constructed using direct sums (in place of infinite direct products). Thus, our problem reduces to showing that the cup product preserves the LieHodge decomposition of $\operatorname{HH}_{\oplus}^{*}(\mathcal{A}, \mathcal{A})$. To prove this, we recall that by definition, $\mathcal{A}$ is a symmetric algebra equipped with a differential which encodes the $L_{\infty}$-structure on $\mathrm{H}_{*}(\mathfrak{a})$. Hence, by the HochschildKostant-Rosenberg theorem, there is a natural linear map $\mathrm{I}_{\mathrm{HKR}}: \mathrm{H}^{*}[\mathcal{V}] \rightarrow \mathrm{HH}_{\oplus}^{*}(\mathcal{A}, \mathcal{A})$, where $\mathrm{H}^{*}[\mathcal{V}]$ denotes the cohomology ring of the algebra $\mathcal{V}=\mathcal{V}(\mathcal{A})$ of polyderivations of $\mathcal{A}$. It is easy to show that the map I IKR preserves Hodge grading; however, in general, it is not a homomorphism of graded algebras. In fact, Kontsevich's (cohomological) version of Duflo's classical theorem, which applies to $\mathcal{A}$ because 
$\mathrm{H}_{*}(\mathfrak{a})$ is finite dimensional, says that to get an algebra isomorphism between $\mathrm{H}^{*}[\mathcal{V}]$ and $\mathrm{HH}_{\oplus}^{*}(\mathcal{A}, \mathcal{A})$, one needs to 'correct' the HKR map by contracting it with a certain canonical cohomology class called the Todd genus. In general, contraction by the Todd genus does not preserve the Hodge grading on $\mathcal{V}$; however, when $\mathrm{H}_{*}(\mathfrak{a})$ is a nilpotent $L_{\infty}$-algebra, we show (see Proposition 4.1) that the Todd genus is actually trivial. ${ }^{2}$ Thus, it turns out that under our assumptions on $\mathfrak{a}$, the HKR map is an isomorphism of graded algebras. Now, since the product on $\mathcal{V}$ obviously preserves the Hodge grading, this completes the proof of Theorem 1.2.

We would like to conclude this introduction by mentioning a well-known analogy between rational homotopy theory and local commutative algebra [1]. The rationally elliptic spaces correspond in commutative algebra to local complete intersection rings, and the $S^{1}$-equivariant homology of free loop spaces to (relative) cyclic homology of local rings. It seems natural to ask whether a result parallel to our Theorem 1.1 holds in commutative algebra. Although one can consider Lie algebra models for local rings (analogues of Quillen models in topology), our 'abstract' Theorem 1.2 does not apply directly to such models, since they are not defined over fields of characteristic 0 .

The paper is organised as follows. In Section 2, we review the Loday-Goodwillie (simplicial) approach to Hodge decompositions and show how the Lie-Hodge decomposition of $\overline{\mathrm{HC}}_{*}(\mathcal{U} \mathfrak{a})$ constructed in [3] arises from this approach. We also construct the Lie-Hodge decomposition on the Hochschild cohomology $\mathrm{HH}^{*}\left(\mathcal{U}_{\mathfrak{a}}, \mathcal{U}_{\mathfrak{a}}\right)$ and prove some technical results needed for our main theorem. In Section 3 we recall definitions and review some known results on derived Poisson structures. The most important result for us (proven in [15]) relates the cyclic derived Poisson structure on $\mathcal{U}_{\mathfrak{a}}$ to the cup product and Gerstenhaber bracket on the Hochschild cohomology of Ua via the Van den Bergh duality. We also show that the Van den Bergh duality is compatible with Lie-Hodge decompositions (Lemma 3.2). Finally, Section 4 contains the proof of Theorem 1.2, as well as its application to string topology.

\section{Notation}

Throughout this paper, $k$ denotes a field of characteristic 0 . All vector spaces, chain complexes and associative and Lie algebras - as well as unadorned multilinear operations, such as $\otimes$, Hom, Sym and others - are defined over $k$. The categories of chain complexes, DG algebras, commutative DG algebras, DG Lie algebras and (conilpotent) DG coalgebras are denoted $\mathrm{Com}_{k}, \mathrm{DGA}_{k}, \mathrm{DGCA}_{k}, \mathrm{DGLA}_{k}$ and $\mathrm{DGC}_{k}$, respectively. The corresponding categories of augmented algebras and coaugmented coalgebras are denoted $\mathrm{DGA}_{k / k}, \mathrm{DGCA}_{k / k}$ and $\mathrm{DGC}_{k / k}$. The Koszul sign rule is tacitly used throughout the paper.

\section{Hodge decompositions}

It is well known that the cyclic homology of any commutative (DG) algebra $A$ has a natural decomposition

$$
\mathrm{HC}_{*}(A)=\bigoplus_{p=0}^{\infty} \mathrm{HC}_{*}^{(p)}(A),
$$

which is usually called the Hodge (or $\lambda$-) decomposition of $\mathrm{HC}_{*}(A)$. Loday [45] gave an elegant explanation of this phenomenon in terms of the classical bar construction $\mathrm{C}_{*}(A)$ of the algebra $A$. Recall that for any associative algebra, $\mathrm{C}_{*}(A)$ is a cyclic module - that is, a functor $\Delta C^{\text {op }} \rightarrow \operatorname{Com}_{k}$ defined on (the opposite of) Connes' cyclic category $\Delta C$. The category $\Delta C^{\text {op }}$ naturally embeds into the category of finite sets Fin, and the theorem of Loday (compare [46, Theorem 6.4.5]) asserts that the cyclic homology of a cyclic module $E_{*}: \Delta C^{\mathrm{op}} \rightarrow \mathrm{Com}_{k}$ admits a direct sum decomposition of the form in equation (2.1) whenever the functor $E_{*}$ extends to Fin - that is, factors through the inclusion $\Delta C^{\text {op }} \hookrightarrow$ Fin. Now, for the cyclic bar construction $E_{*}=\mathrm{C}_{*}(A)$, this happens exactly when $A$ is a commutative algebra (see [46, Proposition 6.4.4]).

\footnotetext{
${ }^{2}$ In the case when $\mathfrak{a}$ is an ordinary finite-dimensional nilpotent Lie algebra, this observation goes back essentially to Duflo's original paper [20] (see also [50]).
} 
Goodwillie (compare [46, Exercise E.6.4.5]) observed that the Hodge decomposition of $\mathrm{HC}_{*}(E)$ may exist in a more general situation: namely, when the functor $E_{*}: \Delta C^{\mathrm{op}} \rightarrow \mathrm{Com}_{k}$ admits an extension $E_{*}^{\Psi}: \Delta \Psi^{\mathrm{op}} \rightarrow \operatorname{Com}_{k}$ to the so-called epicyclic category $\Delta \Psi^{\mathrm{op}}$. The category $\Delta \Psi$ has the same objects as $\Delta C$ but contains extra morphisms which induce the power (Adams) operations on $\mathrm{HC}_{*}(E)$; on the other hand, $\Delta \Psi$ is strictly smaller than Fin. Thus, if $E_{*}: \Delta C \mathrm{op} \rightarrow \mathrm{Com}_{k}$ factors through $\Delta C^{\mathrm{op}} \hookrightarrow \Delta \Psi^{\mathrm{op}}$, then $\mathrm{HC}_{*}(E)$ has a natural Hodge decomposition; furthermore, this decomposition agrees with Loday's when the corresponding epicyclic module $E_{*}^{\Psi}: \Delta \Psi$ op $\rightarrow \operatorname{Com}_{k}$ factors through $\Delta \Psi^{\mathrm{op}} \hookrightarrow$ Fin.

In this section, we show that the cyclic bar construction $\mathrm{C}_{*}(\mathcal{U} \mathfrak{a})$ of the universal enveloping algebra of a (DG) Lie algebra $\mathfrak{a}$ has a natural epicyclic structure (which does not factor through Fin). We prove that the Hodge decomposition of cyclic homology $\overline{\mathrm{HC}}_{*}(\mathcal{U} \mathfrak{a})$ arising from this epicyclic structure coincides with the Lie-Hodge decomposition constructed in [3]. We also establish some properties of Hodge decomposition of Hochschild homology which we will need for the proof of our main theorem.

\subsection{Epicyclic modules and Adams operations}

Let $\Delta$ denote the standard simplicial category whose objects are the finite ordered sets $[n]=\{0<$ $1<2<\cdots<n\}$ and morphisms are the order-preserving maps. The category $\Delta$ is generated by two families of maps $d_{n}^{i}:[n-1] \rightarrow[n]$ (with $0 \leq i \leq n, n \geq 1$ ) and $s_{n}^{j}:[n+1] \rightarrow[n]$ (with $0 \leq j \leq n$, $n \geq 0$ ), called the (co)face and (co)degeneracy maps respectively. These maps satisfy the standard (co)simplicial relations given, for example, in [46, Appendix B.3]. Connes' cyclic category $\Delta C$ is an extension of $\Delta$ that contains - in addition to $d_{n}^{i}$ and $s_{n}^{j}$ - the cyclic maps $\tau_{n}:[n] \rightarrow[n]$ for $n \geq 0$. More generally, for any integer $k \geq 1$, we can define the $k$-cyclic category $\Delta C^{(k)}$ that contains $\Delta$ (and has the same objects as $\Delta$ ) with additional morphisms ${ }_{k} \tau_{n}:[n] \rightarrow[n]$ satisfying

$$
{ }_{k} \tau_{n} \circ d_{n}^{i}=d_{n}^{i-1} \circ{ }_{k} \tau_{n}, \quad{ }_{k} \tau_{n} \circ s_{n}^{j}=s_{n}^{j-1} \circ{ }_{k} \tau_{n}, \quad\left({ }_{k} \tau_{n}\right)^{k(n+1)}=\operatorname{Id}_{[n]} .
$$

There are two natural functors relating $\Delta C^{(k)}$ to $\Delta C \equiv \Delta C^{(1)}$ :

$$
\mathrm{P}^{k}: \Delta C^{(k)} \rightarrow \Delta C, \quad \mathrm{Sd}^{k}: \Delta C^{(k)} \rightarrow \Delta C .
$$

The functor $\mathrm{P}^{k}$ is characterised by the property that its restriction to $\Delta$ is the identity, while $\mathrm{P}^{k}\left({ }_{k} \tau_{n}\right)=\tau_{n}$ for all $n \geq 0$. The functor $\mathrm{Sd}^{k}$ - called the kth edgewise subdivision [48] - is defined by

$$
\operatorname{Sd}^{k}([n]):=[k(n+1)-1]=[n] \sqcup \cdot \stackrel{k}{*} \cdot \sqcup[n],
$$

and on morphisms as

$$
\operatorname{Sd}^{k}(\varphi)=\varphi \sqcup \stackrel{k}{k} \cdot \sqcup \varphi, \text { for } \varphi \in \operatorname{Mor}(\Delta), \quad \operatorname{Sd}^{k}\left({ }_{k} \tau_{n}\right)=\tau_{k(n+1)-1},
$$

where the notation $[n] \sqcup . k \cdot \sqcup[n]$ means taking the disjoint union of $k$ copies of the set $[n]$, and $\varphi \sqcup \stackrel{k}{*} \cdot \sqcup \varphi$ means applying the map $\varphi$ to each of these copies separately.

Now, the epicyclic category $\Delta \Psi$ [12] is the extension of $\Delta C$ (i.e., $\Delta C \subset \Delta \Psi$ ), which - in addition to the morphisms $\left\{d_{n}^{i}\right\},\left\{s_{n}^{j}\right\}$ and $\left\{\tau_{n}\right\}$ generating $\Delta C$ - contains a family of morphisms

$$
\pi_{n}^{k}:[k(n+1)-1] \rightarrow[n], \quad \forall n \geq 0, k \geq 1,
$$

called the (co)power maps. These maps are characterised by the property that $\pi_{*}^{k}: \mathrm{Sd}^{k} \rightarrow \mathrm{P}^{k}$ define natural transformations of functors $\Delta C^{(k)} \rightarrow \Delta C \hookrightarrow \Delta \Psi$ for all $k \geq 1$ and, in addition, satisfy the 
relations

$$
\pi_{n}^{1}=\operatorname{Id}_{[n]}, \quad \pi_{n}^{l} \circ \pi_{l(n+1)-1}^{k}=\pi_{n}^{k l} .
$$

If $\mathcal{C}$ is a category, an epicyclic object in $\mathcal{C}$ is, by definition, a functor $X: \Delta \Psi^{\mathrm{op}} \rightarrow \mathcal{C}$; we will write $\mathcal{C}_{\Delta \Psi}$ for the category of such functors, with morphisms being the natural transformations. Note that giving an epicyclic object in $\mathcal{C}$ is equivalent to giving a cyclic object $X: \Delta C^{\mathrm{op}} \rightarrow \mathcal{C}$ together with a family of morphisms in $\Delta C^{(k)}(k \geq 1)$ :

$$
p_{*}^{k}(X): \mathrm{P}_{*}^{k}(X) \rightarrow \operatorname{Sd}_{*}^{k}(X), \quad p_{n}^{k}: X_{n} \rightarrow X_{k(n+1)-1},
$$

satisfying

$$
p_{*}^{1}=\mathrm{Id}, \quad p_{*}^{k} \circ p_{*}^{l}=p_{*}^{k l},
$$

where $\mathrm{P}_{*}^{k}(X)$ and $\mathrm{Sd}_{*}^{k}(X)$ are the $k$-cyclic objects $\left(\Delta C^{(k)}\right)^{\text {op }} \rightarrow \mathcal{C}$ defined by $\mathrm{P}_{*}^{k}(X):=X \circ \mathrm{P}^{k}$ and $\mathrm{Sd}_{*}^{k}(X):=X \circ \mathrm{Sd}^{k}$ (see (2.2)). It is a classical observation (due to Connes) that when $\mathcal{C}=$ Set, the geometric realisation $|X|$ of any cyclic set $X: \Delta C^{\text {op }} \rightarrow$ Set caries a natural $S^{1}$-action (see [46, Theorem 7.1.4]). In a similar way, if $X$ is an epicyclic set, then in addition to the $S^{1}$-action its realisation $|X|$ carries power operations which induce Adams operations on the $S^{1}$-equivariant homology $\mathrm{H}_{*}^{S^{1}}(|X|)$ of $|X|[12$, Theorem $\mathrm{A}]$ ). We will look at an algebraic (chain) version of this construction.

Recall that to any associative unital (DG) $k$-algebra $A$, one can naturally attach a cyclic module

$$
\mathrm{C}_{*}(A): \Delta C^{\mathrm{op}} \rightarrow \mathrm{Com}_{k}, \quad[n] \mapsto A^{\otimes(n+1)},
$$

called the cyclic bar construction [46, Proposition 2.5.4]. Our main observation in this section is the following:

Proposition 2.1. If $A$ is a cocommutative $(D G)$ Hopf algebra, then $\mathrm{C}_{*}(A)$ carries a natural epicyclic structure.

To prove Proposition 2.1, we need to extend the functor (2.4) to the epicyclic category - that is, construct a functor $\mathrm{C}_{*}^{\Psi}(A): \Delta \Psi$ op $\rightarrow \operatorname{Com}_{k}$ such that $\left.\mathrm{C}_{*}^{\Psi}(A)\right|_{\Delta C^{\mathrm{op}}}=\mathrm{C}_{*}(A)$. This can be done directly by defining the structure maps (2.3) and verifying the required relations. We will give a more conceptual construction of $\mathrm{C}_{*}^{\Psi}(A)$ that relies on the well-known fact that the cocommutative Hopf algebras are the group objects in the category of cocommutative coalgebras.

Let $(5$ denote (the skeleton of) the category of finitely generated free groups: thus, the objects of (5) are the free groups $\langle n\rangle=\mathbb{F}_{n}$, one for each cardinality $n \geq 0$, and the morphisms $\langle n\rangle \rightarrow\langle m\rangle$ are arbitrary group homomorphisms $\mathbb{F}_{n} \rightarrow \mathbb{F}_{m}$. The category $\mathbf{6}$ carries a (strict) monoidal structure with product $\langle n\rangle *\langle m\rangle=\langle n+m\rangle$ for all $n, m \geq 0$. The category of all (discrete) groups $\mathrm{Gr}$ can then be described as the category $\operatorname{Set}_{\mathfrak{W}}^{\otimes}$ of strict monoidal functors ${\left(5^{\circ p}\right.}^{\text {op }} \rightarrow$ Set with values in Set equipped with the usual (cartesian) monoidal structure: the equivalence $\mathrm{Gr} \stackrel{\sim}{\rightarrow} \operatorname{Set}_{\mathfrak{G}}^{\otimes}$ is given by the Yoneda functor $G \mapsto \underline{G}:=\operatorname{Hom}_{\mathrm{Gr}}(-, G)$ restricted to the subcategory $\mathfrak{( b} \subset$ Gr. Now, it is known and easy to check (see, e.g., [9] and [12, Example 1.3]) that the cyclic nerve $N_{*}^{\text {cyc }}(G):=$ $\left\{G^{n+1}\right\}_{n \geq 0}$ of any discrete group $G$ carries a canonical epicyclic structure with power maps (2.3) given by

$$
p_{n}^{k}: G^{n+1} \rightarrow G^{k(n+1)}, \quad\left(g_{0}, \ldots, g_{n}\right) \mapsto\left(g_{0}, \ldots, g_{n} ; . k ; g_{0}, \ldots, g_{n}\right) .
$$

Thus we have a well-defined functor $N_{*}^{\text {cyc }}:$ Gr $\rightarrow \operatorname{Set}_{\Delta \Psi}$. If we identify $\mathrm{Gr} \cong \operatorname{Set}_{\mathfrak{G}}^{\otimes}$ via $G \mapsto \underline{G}$ as before, then $N_{*}^{\text {cyc }}$ is simply the pullback functor $\Psi^{*}: \operatorname{Set}_{\mathfrak{G}}^{\otimes} \rightarrow \operatorname{Set}_{\Delta \Psi}$ for a natural map in Cat:

$$
\Psi: \Delta \Psi \rightarrow \mathfrak{6} .
$$


Explicitly, the functor (2.5) is defined on objects by $\Psi([n])=\langle n+1\rangle=\mathbb{F}\left\langle x_{0}, \ldots, x_{n}\right\rangle$ and on morphisms by the following formulas:

$$
\begin{aligned}
& \Psi\left(d_{n}^{i}\right):\langle n\rangle \rightarrow\langle n+1\rangle, \quad\left(x_{0}, \ldots, x_{n-1}\right) \mapsto \begin{cases}\left(x_{0}, \ldots, x_{i-1}, x_{i} x_{i+1}, \ldots, x_{n}\right), & i<n, \\
\left(x_{n} x_{0}, x_{1}, \ldots, x_{n-1}\right), & i=n,\end{cases} \\
& \Psi\left(s_{n}^{j}\right):\langle n+2\rangle \rightarrow\langle n+1\rangle, \quad\left(x_{0}, \ldots, x_{n+1}\right) \mapsto\left(x_{0}, \ldots, x_{j}, 1, x_{j+1}, \ldots, x_{n}\right), \\
& \Psi\left(\tau_{n}\right):\langle n+1\rangle \rightarrow\langle n+1\rangle, \quad\left(x_{0}, \ldots, x_{n}\right) \mapsto\left(x_{n}, x_{0}, x_{1}, \ldots, x_{n-1}\right), \\
& \Psi\left(\pi_{n}^{k}\right):\langle k(n+1)\rangle \rightarrow\langle n+1\rangle, \quad x_{m} \mapsto x_{\bar{m}}, \quad m=0,1, \ldots, k(n+1)-1 .
\end{aligned}
$$

where $\bar{m}$ denotes the remainder of $m$ modulo $n+1$.

Proof (of Proposition 2.1). The category of cocommutative (DG) Hopf algebras is equivalent to the category $\operatorname{Com}_{\mathfrak{G}}^{\otimes}$ of strict monoidal functors $\mathbf{5}^{\text {op }} \rightarrow \mathrm{Com}_{k}$, with an algebra $A$ corresponding to the functor $\underline{A}:\left(^{\circ o p} \rightarrow \operatorname{Com}_{k},\langle n\rangle \mapsto A^{\otimes n}\right.$ (see, e.g., [51]). Now, the epicyclic module $C_{*}^{\Psi}(A)$ associated to $A$ is simply given by the composition

$$
\mathrm{C}_{*}^{\Psi}(A): \Delta \Psi^{\mathrm{op}} \stackrel{\Psi \text { op }}{\longrightarrow} \mathfrak{G}^{\mathrm{op}} \stackrel{A}{\longrightarrow} \mathrm{Com}_{k}, \quad[n] \mapsto A^{\otimes n+1},
$$

where $\Psi^{\mathrm{op}}$ is the opposite functor of the functor (2.5). Note that by the construction of $\Psi$, the restriction of $C_{*}^{\Psi}(A)$ to $\Delta C^{\mathrm{op}} \subset \Delta \Psi^{\mathrm{op}}$ coincides with the cyclic bar construction (2.4) associated to $A$ as an algebra.

Remark. The functor $\Psi$ does not factor through Fin nor its opposite category Fin ${ }^{\text {op }}$. Thus, unlike in [45], our epicyclic bar construction $C_{*}^{\Psi}(A): \Delta \Psi$ op $\rightarrow \operatorname{Com}_{k}$ does not extend to all finite sets. To avoid confusion, we note that there is a different functor $\Delta \Psi \rightarrow \mathfrak{G}$ that factors naturally through Fin: to construct it, consider the composition

$$
\Delta C \stackrel{\sim}{\rightarrow} \Delta C^{\mathrm{op}} \stackrel{S_{*}^{1}}{\rightarrow} \text { Fin }
$$

where the first arrow is Connes' cyclic duality identifying the cyclic category with its opposite [46, Proposition 6.1.11] and the second is the standard cyclic model of the circle $S^{1}$. It is known and easy to check (see, e.g., [46, Exercise E.6.4.4]) that the functor (2.6) extends to $\Delta \Psi$, giving a map $\Delta \Psi \rightarrow$ Fin. Now, combining this last map with the free group functor $\mathbb{F}:$ Set $\rightarrow \mathrm{Gr}$, we define

$$
\Phi: \Delta \Psi \rightarrow \text { Fin } \stackrel{\mathbb{F}}{\rightarrow} \mathfrak{G} .
$$

When restricted to $\Delta$, the functor (2.6) coincides with the natural inclusion $\Delta \hookrightarrow$ Fin which identifies $[n]=\{0,1,2, \ldots, n\}[46$, Remark 6.4.3]. Hence, on objects, the functor $\Phi$ agrees with $\Psi$, mapping $[n] \rightarrow\langle n+1\rangle$. However, the values of $\Phi$ on morphisms are quite different from those of $\Psi$. In fact, the group homomorphisms $\Phi\left(d_{n}^{i}\right):\langle n\rangle \rightarrow\langle n+1\rangle$ corresponding to the coface maps $d_{n}^{i}:[n-1] \rightarrow[n]$ are given by $x_{k} \mapsto x_{k}$ for $k<i$ and $x_{k} \mapsto x_{k+1}$ for $k \geq i$, while for the codegeneracy maps $s_{n}^{j}:[n+1] \rightarrow[n]$ we have $\Phi\left(s_{n}^{j}\right):\langle n+2\rangle \rightarrow\langle n+1\rangle, x_{k} \mapsto x_{k}$, for $k \leq j$ and $x_{k} \mapsto x_{k-1}$ for $k>j$. This shows that for a cocommutative Hopf algebra $A$, the cyclic module $\Phi^{*}(\underline{A}):=\underline{A} \circ \Phi^{\mathrm{op}}: \Delta C^{\mathrm{op}} \rightarrow \mathrm{Com}_{k}$ depends only on the coalgebra structure of $A$, and hence it is entirely different from $\mathrm{C}_{*}(A)$. By Loday [45], the cyclic homology of $\Phi^{*}(\underline{A})$ admits a direct decomposition which is simply a formal (coalgebra) dual to the classical Hodge decomposition for commutative algebras (2.1).

It follows from Proposition 2.1 that if $A$ is a cocommutative Hopf algebra, the cyclic module $\mathrm{C}_{*}(A)$ is equipped with extra power operations (see (2.3)) given by simplicial maps:

$$
p_{*}^{k}(A): \mathrm{C}_{*}(A) \rightarrow \mathrm{Sd}^{k}\left[\mathrm{C}_{*}(A)\right], \quad k \geq 1 .
$$


To describe these maps we need first to identify their targets $\operatorname{Sd}^{k}\left[\mathrm{C}_{*}(A)\right]$. Recall that for any (DG) algebra $A$ and any (DG) $A$-bimodule $M$, one can define a simplicial (complex of) module(s) $\mathrm{C}_{*}(A, M)=\left\{\mathrm{C}_{n}(A, M)=M \otimes A^{\otimes n}\right\}_{n \geq 0}$ whose homology is the Hochschild homology $\mathrm{HH}_{*}(A, M)$. In particular, for $M=A$ we have $\mathrm{C}_{*}(A, A)=\mathrm{C}_{*}(A)$. We will use this construction for the bimodule ${ }_{t} A^{\otimes k}$ over the algebra $A^{\otimes k}$, where the left $A^{\otimes k}$-module structure is twisted by a cyclic permutation that is,

$$
\left(a_{1} \otimes \cdots \otimes a_{k}\right) \cdot\left(b_{1} \otimes \cdots \otimes b_{k}\right)=a_{k} b_{1} \otimes a_{1} b_{2} \otimes \cdots \otimes a_{k-1} b_{k}
$$

\section{Lemma 2.1.}

(a) For every $k \geq 1$, there is an isomorphism of simplicial modules

$$
\operatorname{Sd}^{k}\left[\mathrm{C}_{*}(A)\right] \stackrel{\sim}{\rightarrow} \mathrm{C}_{*}\left(A^{\otimes k},{ }_{t} A^{\otimes k}\right)
$$

given (in simplicial degree n) by 'transposition of matrices':

$$
\begin{gathered}
A^{\otimes(n+1)} \otimes \cdot \stackrel{k}{*} \otimes A^{\otimes(n+1)} \rightarrow A^{\otimes k} \otimes \stackrel{n+1}{*} \otimes A^{\otimes k}, \\
\left(\begin{array}{cccc}
a_{0} & a_{n+1} & \cdots & a_{(k-1)(n+1)} \\
a_{1} & a_{n+2} & \cdots & a_{(k-1)(n+1)+1} \\
\vdots & \vdots & \ddots & \vdots \\
a_{n} & a_{2 n+1} & \cdots & a_{k(n+1)-1}
\end{array}\right) \mapsto\left(\begin{array}{cccc}
a_{0} & a_{1} & \cdots & a_{n} \\
a_{n+1} & a_{n+2} & \cdots & a_{2 n+1} \\
\vdots & \vdots & \ddots & \vdots \\
a_{(k-1)(n+1)} & a_{(k-1)(n+1)+1} & \cdots & a_{k(n+1)-1}
\end{array}\right),
\end{gathered}
$$

where the elements of the tensor powers $A^{\otimes(n+1)}$ and $A^{\otimes k}$ are represented as matrix columns.

(b) With identification (2.8), the power maps (2.7) are given by

$$
p_{n}^{k}: A^{\otimes(n+1)} \rightarrow A^{\otimes k} \otimes \stackrel{(n+1)}{\cdots} \otimes A^{\otimes k}, \quad a_{0} \otimes \cdots \otimes a_{n} \mapsto \Delta^{k}\left(a_{0}\right) \otimes \cdots \otimes \Delta^{k}\left(a_{n}\right),
$$

where $\Delta^{k}: A \rightarrow A^{\otimes k}$ is the $k$-iterated coproduct on $A$.

Proof. (a) Straightforward verification. We leave it as an exercise to the reader. $(b)$ By definition, the maps $p_{n}^{k}$ are the images of the generating morphisms $\pi_{n}^{k}:[(n+1) k-1] \rightarrow[n]$ of the category $\Delta \Psi$. Under the functor $\Psi$ (see (2.5)), these morphisms correspond to the folding maps

$$
\nabla_{n+1}^{k}:\langle k(n+1)\rangle=\langle n+1\rangle * \cdot k \cdot *\langle n+1\rangle \rightarrow\langle n+1\rangle,
$$

which act as identities $\operatorname{Id}_{\langle n+1\rangle}$ on each copy of the free group $\langle n+1\rangle$ in $\langle k(n+1)\rangle$. Now, it is easy to see that the maps $\nabla_{n+1}^{k}$ factor in $(5$ as

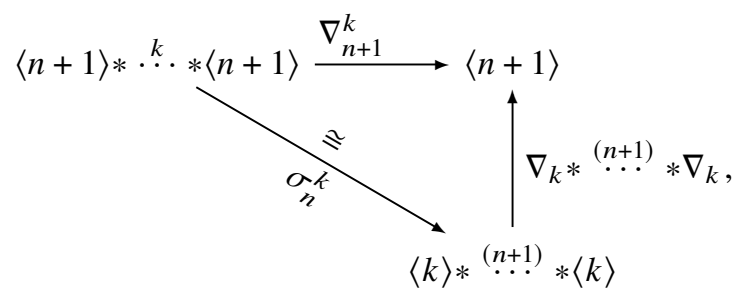


where $\nabla_{k}:\langle k\rangle=\langle 1\rangle * \cdots *\langle 1\rangle \rightarrow\langle 1\rangle$ is the $k$-folding map for $\langle 1\rangle \in \mathfrak{5}$ and $\sigma_{n}^{k}$ is the isomorphism of free groups given by the transposition

$$
\left(\begin{array}{cccc}
x_{0} & x_{n+1} & \cdots & x_{(k-1)(n+1)} \\
x_{1} & x_{n+2} & \cdots & x_{(k-1)(n+1)+1} \\
\vdots & \vdots & \ddots & \vdots \\
x_{n} & x_{2 n+1} & \cdots & x_{k(n+1)-1}
\end{array}\right) \mapsto\left(\begin{array}{cccc}
x_{0} & x_{1} & \cdots & x_{n} \\
x_{n+1} & x_{n+2} & \cdots & x_{2 n+1} \\
\vdots & \vdots & \ddots & \vdots \\
x_{(k-1)(n+1)} & x_{(k-1)(n+1)+1} & \cdots & x_{k(n+1)-1}
\end{array}\right)
$$

where the matrix columns represent the generators of the corresponding factors of the free products. Since under the functor $\underline{A}: \mathfrak{G}^{\text {op }} \rightarrow \operatorname{Com}_{k}$ the folding maps $\nabla_{k}:\langle k\rangle \rightarrow\langle 1\rangle$ correspond exactly to the $k$-iterated coproducts $\Delta^{k}: A \rightarrow A^{\otimes k}$, the claim of part (b) follows.

Finally, using Lemma 2.1 we describe the Adams operations induced by the power maps (2.7) on the cyclic homology of a cocommutative DG Hopf algebra. Let $R$ be a cocommutative DG Hopf algebra which is cofibrant as an object in $\mathrm{DGA}_{k / k}$. Let $R_{\natural}:=R /(k+[R, R])$ denote the cyclic construction on $R$ which computes - by a theorem of Feigin and Tsygan (see, e.g., [5, 22]) - the (reduced) cyclic homology $\overline{\mathrm{HC}}_{*}(R)$ of $R$ viewed as an associative DG algebra. ${ }^{3}$ Then, applying Lemma 2.1 to the epicyclic module $\mathrm{C}_{*}(R)$, we get the commutative diagram

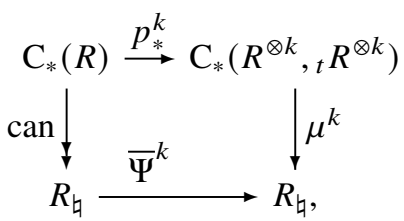

where can is the canonical projection onto $\pi_{0} \mathrm{C}_{*}(R) \cong R_{\natural}$ and $\mu^{k}$ is the composition of the natural map $\pi_{0}: \mathrm{C}_{*}\left(R^{\otimes k},{ }_{t} R^{\otimes k}\right) \rightarrow\left({ }_{t} R^{\otimes k}\right)_{\natural}$ with the map $\left({ }_{t} R^{\otimes k}\right)_{\natural} \stackrel{\bar{\mu}}{\rightarrow} R_{\natural}$ induced by iterated multiplication on $R$. It follows from Lemma 2.1(b) that the maps $\bar{\Psi}^{k}: R_{\natural} \rightarrow R_{\natural}$ in diagram (2.9) are induced by the compositions

$$
\Psi^{k}: R \stackrel{\Delta^{k}}{\rightarrow} R^{\otimes k} \stackrel{\mu}{\rightarrow} R, \quad k \geq 1
$$

Thus, we conclude the following:

Corollary 2.1. For any cocommutative DG Hopf algebra $R$ which is cofibrant in $\mathrm{DGA}_{k / k}$, the Adams operations on $\overline{\mathrm{HC}}_{*}(R)$ coming from the epicyclic structure on $\mathrm{C}_{*}(R)$ are induced by the maps (2.10).

In the next section, we will give a different construction of these Adams operations in terms of derived functors, following [3].

\subsection{Lie-Hodge decomposition}

Given a Lie algebra $\mathfrak{a}$ over $k$, we consider the symmetric ad-invariant $k$-multilinear forms on $\mathfrak{a}$ of (fixed) degree $p \geq 1$. Every such form is induced from the universal one: $\mathfrak{a} \times \mathfrak{a} \times \cdots \times \mathfrak{a} \rightarrow \lambda^{(p)}(\mathfrak{a})$, which takes its values in the space $\lambda^{(p)}(\mathfrak{a})$ of coinvariants of the adjoint representation of $\mathfrak{a}$ in $\operatorname{Sym}^{p}(\mathfrak{a})$. The assignment $\mathfrak{a} \mapsto \lambda^{(p)}(\mathfrak{a})$ defines a (nonadditive) functor on the category of Lie algebras that naturally extends to the category of DG Lie algebras:

$$
\lambda^{(p)}: \operatorname{DGLA}_{k} \rightarrow \operatorname{Com}_{k}, \quad \mathfrak{a} \mapsto \operatorname{Sym}^{p}(\mathfrak{a}) /\left[\mathfrak{a}, \operatorname{Sym}^{p}(\mathfrak{a})\right] .
$$

${ }^{3}$ Recall that the cyclic homology of an arbitrary (not necessarily cofibrant) DG algebra $R$ can be defined explicitly as the homology of (the total complex of) Connes' bicomplex of $R$ (see, e.g., [46, Section 5.3.3]). 
The functor (2.11) does not preserve quasi-isomorphisms and hence does not descend to the homotopy category $\operatorname{Ho}\left(\right.$ DGLA $\left._{k}\right)$. To remedy this problem, we replace $\lambda^{(p)}$ by its (left) derived functor

$$
\boldsymbol{L} \lambda^{(p)}: \operatorname{Ho}\left(\mathrm{DGLA}_{k}\right) \rightarrow \mathcal{D}(k),
$$

which takes its values in the derived category $\mathcal{D}(k)$ of chain complexes. We write $\mathrm{HC}_{*}^{(p)}(\mathfrak{a})$ for the homology of $\boldsymbol{L} \lambda^{(p)}(\mathfrak{a})$, and call it the Lie-Hodge homology of $\mathfrak{a}$.

For $p=1$, the functor $\lambda^{(1)}$ is just the abelianisation of Lie algebras; in this case, the existence of $\boldsymbol{L} \lambda^{(1)}$ follows from Quillen's general theory [52, Chapter II, §5]), and $\mathrm{HC}_{*}^{(1)}(\mathfrak{a})$ coincides (up to shift in degree) with the classical Chevalley-Eilenberg homology $\mathrm{H}_{*}(\mathfrak{a}, k)$ of the Lie algebra $\mathfrak{a}$. For $p=2$, the functor $\lambda^{(2)}$ was introduced by Drinfeld [19]; the existence of $\boldsymbol{L} \lambda^{(2)}$ was established by Getzler and Kapranov [27], who suggested that $\mathrm{HC}_{*}^{(2)}(\mathfrak{a})$ should be viewed as an analogue of cyclic homology for Lie algebras. The existence of $\boldsymbol{L} \lambda^{(p)}$ for arbitrary $p$ was established in [3, Section 7].

Next, consider the functor

$$
(-)_{\text {! }}: \mathrm{DGA}_{k / k} \rightarrow \mathrm{Com}_{k}, \quad R \mapsto R /(k+[R, R]),
$$

which is called the cyclic functor on associative DG algebras (compare [22]). Observe that each $\lambda^{(p)}$ comes together with a natural transformation to the composite functor

$$
\mathcal{U}_{\natural}: \operatorname{DGLA}_{k} \stackrel{\mathrm{u}}{\longrightarrow} \mathrm{DGA}_{k / k} \stackrel{(-)_{\natural}}{\longrightarrow} \operatorname{Com}_{k},
$$

where $\mathcal{U}$ is the universal enveloping algebra functor on the category of (DG) Lie algebras. The natural transformations $\lambda^{(p)} \rightarrow \mathcal{U}_{\natural}$ are induced by the symmetrisation maps

$$
\operatorname{Sym}^{p}(\mathfrak{a}) \rightarrow \mathcal{U} \mathfrak{a}, \quad x_{1} x_{2} \cdots x_{p} \mapsto \frac{1}{p !} \sum_{\sigma \in \Sigma_{p}} \pm x_{\sigma(1)} \cdot x_{\sigma(2)} \cdots x_{\sigma(p)},
$$

which by the Poincaré-Birkhoff-Witt theorem assemble to an isomorphism of DG a-modules $\operatorname{Sym}_{k}(\mathfrak{a}) \cong \mathcal{U}_{\mathfrak{a}}$. From this, it follows that $\lambda^{(p)} \rightarrow \mathcal{U}_{\natural}$ give an isomorphism of functors

$$
\bigoplus_{p=1}^{\infty} \lambda^{(p)} \cong \mathcal{U}_{\natural}
$$

On the other hand, by a theorem of Feigin and Tsygan [22] (see also [5]), the functor $(-)_{\natural}$ has a left derived functor $\boldsymbol{L}(-)_{\natural}: \operatorname{Ho}\left(\mathrm{DGA}_{k / k}\right) \rightarrow \mathcal{D}(k)$ that computes the reduced cyclic homology $\overline{\mathrm{HC}}_{*}(R)$ of an associative algebra $R \in \mathrm{DGA}_{k / k}$. Since $\mathcal{U}$ preserves quasi-isomorphisms and maps cofibrant DG Lie algebras to cofibrant DG associative algebras, the isomorphism (2.14) induces an isomorphism of derived functors from $\mathrm{Ho}\left(\mathrm{DGLA}_{k}\right)$ to $\mathcal{D}(k)$ :

$$
\bigoplus_{p=1}^{\infty} \boldsymbol{L} \lambda^{(p)} \cong \boldsymbol{L}(-)_{\natural} \circ \mathcal{U}
$$

At the level of homology, the isomorphism (2.15) yields the direct decomposition (compare [3, Theorem 7.2])

$$
\bigoplus_{p=1}^{\infty} \mathrm{HC}_{*}^{(p)}(\mathfrak{a}) \cong \overline{\mathrm{HC}}_{*}(\mathcal{U} \mathfrak{a})
$$

To state the main theorem of this section, we recall that the universal enveloping algebra Ua of a (DG) Lie algebra $\mathfrak{a}$ has the natural structure of a cocommutative (DG) Hopf algebra. By Proposition 2.1, the 
associated simplicial module $\mathrm{C}_{*}(\mathcal{U} \mathfrak{a})$ carries therefore an epicyclic structure. We write $\bar{\Psi}^{k}: \overline{\mathrm{HC}}_{*}\left(\mathcal{U}_{\mathfrak{a}}\right) \rightarrow$ $\overline{\mathrm{HC}}_{*}(\mathcal{U} \mathfrak{a}), k \geq 1$, for the Adams operations induced by this structure.

Theorem 2.1. For every $p \geq 2$, the Lie-Hodge homology $\mathrm{HC}_{*}^{(p)}(\mathfrak{a})$ is the common (graded) eigenspace of the operators $\bar{\Psi}^{k}$ corresponding to the eigenvalues $k^{p}, k \geq 1$.

Proof. Without loss of generality, we may assume that $\mathfrak{a}$ is a cofibrant DG Lie algebra. Then, as already explained, $R=U_{\mathfrak{a}}$ is a cocommutative DG Hopf algebra which is cofibrant as an associative DG algebra. By Corollary 2.1, the Adams operations are induced by the maps $\mu_{k} \circ \Delta^{k}: R \rightarrow R$, where $\Delta^{k}: R \rightarrow R^{\otimes k}$ is the $k$-iterated coproduct on $R$ and $\mu_{k}: R^{\otimes k} \rightarrow R$ is the $k$-iterated product. The theorem follows now from [3, Proposition 7.5 and Corollary 7.7], which show that the very same Adams operations $\bar{\Psi}^{k}$ arise from the derived functors $\boldsymbol{L} \lambda^{(p)}$.

Theorem 2.1 implies that the Lie-Hodge decomposition (2.16) arises from the natural epicyclic structure on $\mathrm{C}_{*}\left(\mathcal{U}_{\mathfrak{a}}\right)$ given in Proposition 2.1.

\subsection{Hodge decomposition of Hochschild homology}

The decomposition (2.16) also extends to (reduced) Hochschild homology (see [7, Section 2.1]):

$$
\overline{\mathrm{HH}}_{*}(\mathcal{U} \mathfrak{a}) \cong \bigoplus_{p=0}^{\infty} \mathrm{HH}_{*}^{(p)}(\mathfrak{a})
$$

Recall that there is a natural isomorphism $\overline{\mathrm{HH}}_{*}(\mathcal{U} \mathfrak{a}) \cong \mathrm{H}_{*}(\mathfrak{a} ; \operatorname{Sym}(\mathfrak{a}))$ [46, Theorem 3.3.2]: under this isomorphism, the summand $\mathrm{HH}^{(p)}(\mathfrak{a})$ is identified with $\mathrm{H}_{*}\left(\mathfrak{a} ; \operatorname{Sym}^{p}(\mathfrak{a})\right)$. The Connes periodicity sequence for $\mathcal{U}_{\mathfrak{a}}$ decomposes into a direct sum of Hodge components: the summand of Hodge degree $p$ is given by the long exact sequence [7, Theorem 2.2]

$$
\cdots \stackrel{S}{\rightarrow} \mathrm{HC}_{n-1}^{(p+1)}(\mathfrak{a}) \stackrel{B}{\rightarrow} \mathrm{HH}_{n}^{(p)}(\mathfrak{a}) \stackrel{I}{\rightarrow} \mathrm{HC}_{n}^{(p)}(\mathfrak{a}) \stackrel{S}{\rightarrow} \mathrm{HC}_{n-2}^{(p+1)}(\mathfrak{a}) \rightarrow \cdots
$$

Next we shall show that the Hochschild cohomology $\mathrm{HH}^{*}\left(\mathcal{U}_{\mathfrak{a}}, \mathcal{U}_{\mathfrak{a}}\right)$ has a similar Hodge decomposition. Recall that $A \in \mathrm{DGA}_{k / k}$ is Koszul dual to $C \in \mathrm{DGC}_{k / k}$ if there is a quasi-isomorphism of DG algebras $R:=\mathbf{\Omega}(C) \stackrel{\sim}{\rightarrow} A$, where $\boldsymbol{\Omega}(C)$ denotes the (associative) cobar construction of $C$. Assume that $A$ is Koszul dual to $C$. Let $\iota: C \rightarrow R$ denote the universal twisting cochain. Further recall that given a twisting $\tau: C \rightarrow A$, there is a convolution algebra $\operatorname{Hom}^{\tau}(C, A)$ with twisted differential $d_{\text {Hom }(C, A)}+[\tau,-]$. The following proposition is well known (see, e.g., [49, Theorem 1.1]):

Proposition 2.2. There is an isomorphism of graded $k$-algebras

$$
\mathrm{HH}^{*}(A, A) \cong \mathrm{H}_{-*}\left[\operatorname{Hom}^{\iota}(C, R)\right]
$$

Recall that for a DG algebra $E$, the Hochschild cochain algebra $C^{*}(E, E)$, with product given by the cup product, is isomorphic to $\Pi_{n \geq 0} \operatorname{Hom}\left(\bar{E}[1]^{\otimes n}, E\right)$ as a graded algebra. The graded subspace $\oplus_{n \geq 0} \operatorname{Hom}\left(\bar{E}[1]^{\otimes n}, E\right)$ is a (DG) Gerstenhaber subalgebra of $\mathrm{C}^{*}(E, E)$, which we shall denote by $\mathrm{C}_{\oplus}^{*}(E, E)$. Let $\mathrm{HH}_{\oplus}^{*}(E, E)$ denote the corresponding cohomology.

Corollary 2.2. If $C$ is finite dimensional and $E:=C^{*}$ is the graded linear dual of $C$, then there is an isomorphism of algebras

$$
\mathrm{HH}^{*}(A, A) \cong \mathrm{HH}_{\oplus}^{*}(E, E) .
$$


Proof. Since $C$ is finite dimensional,

$$
\operatorname{Hom}^{\iota}(C, R) \cong \bigoplus_{n \geq 0} \operatorname{Hom}\left(C, \bar{C}[-1]^{\otimes n}\right) \cong \bigoplus_{n \geq 0} \operatorname{Hom}\left(\bar{E}[1]^{\otimes n}, E\right) .
$$

Thus $\operatorname{Hom}^{\iota}(C, R)$ - viewed as a cochain complex by inverting degrees - is isomorphic to the (normalised) Hochschild cochain complex $\mathrm{C}_{\oplus}^{*}(E, E)$ as a graded vector space. Is is easy to verify that the identification turns the differential on $\operatorname{Hom}^{\iota}(C, R)$ into the Hochschild differential on $\mathrm{C}_{\oplus}^{*}(E, E)$ and the convolution product on $\operatorname{Hom}^{\iota}(C, R)$ into the cup product on $\mathrm{C}_{\oplus}^{*}(E, E)$. By Proposition 2.2, there is an isomorphism of associative algebras $\mathrm{HH}^{*}(A, A) \cong \mathrm{HH}_{\oplus}^{*}(E, E)$.

Let $\mathfrak{a} \in \mathrm{DGLA}_{k}$ be Koszul dual to $C \in \mathrm{DGCC}_{k / k}$. This is equivalent to the existence of a quasiisomorphism $\mathcal{L}:=\boldsymbol{\Omega}_{\text {Comm }}(C) \stackrel{\sim}{\rightarrow} \mathfrak{a}$, where $\boldsymbol{\Omega}_{\text {Comm }}(C)$ denotes the (Lie) cobar construction of $C$. Define $R:=\Omega C$ and let $\iota: C \rightarrow R$ be the universal twisting cochain. It is easy to verify that $R \cong \mathcal{U L}$. Since the image of $\iota$ lies in $\mathcal{L} \subset R$, we may view $\iota$ as a twisting cochain from $C$ to $\mathcal{L}$ as well. Let $R^{(p)}$ denote the image of $\operatorname{Sym}^{p}(\mathcal{L})$ in $R$ under the symmetrisation map (2.13). The adjoint action of $\mathcal{L}$ on $R$ induces an action of $\operatorname{Hom}(C, \mathcal{L})$ on $\operatorname{Hom}(C, R)$ : indeed, viewing $\mathcal{L}$ as a Lie subalgebra of $R$, we can consider $[\alpha, f] \in \operatorname{Hom}(C, R)$ for $\alpha \in \operatorname{Hom}(C, \mathcal{L})$ and $f \in \operatorname{Hom}(C, R)$. This action equips $\operatorname{Hom}^{\iota}(C, R)$ with the structure of a Lie module over the DG Lie algebra $\operatorname{Hom}^{\iota}(C, \mathcal{L})$. Further assume that $C$ is finite dimensional.

Theorem 2.2. The natural inclusions $R^{(p)} \hookrightarrow R$ induce a direct sum decomposition of $D G \operatorname{Hom}^{\iota}(C, \mathcal{L})$ modules:

$$
\operatorname{Hom}^{\iota}(C, R) \cong \bigoplus_{p=0}^{\infty} \operatorname{Hom}^{\iota}\left(C, R^{(p)}\right)
$$

As a consequence,

$$
\mathrm{HH}^{*}(\mathcal{U} \mathfrak{a}, \mathcal{U} \mathfrak{a}) \cong \bigoplus_{p=0}^{\infty} \mathrm{H}^{*}\left(\mathfrak{a} ; \operatorname{Sym}^{p}(\mathfrak{a})\right)
$$

Proof. There is an isomorphism of $\mathcal{L}$-modules $R \cong \oplus_{p=0}^{\infty} R^{(p)}$. Since $C$ is finite dimensional,

$$
\operatorname{Hom}(C, R) \cong \bigoplus_{p=0}^{\infty} \operatorname{Hom}\left(C, R^{(p)}\right)
$$

as graded vector spaces. It remains to check that if $\alpha \in \operatorname{Hom}(C, \mathcal{L})$ and $f \in \operatorname{Hom}\left(C, R^{(p)}\right)$, then $[\alpha, f] \in \operatorname{Hom}\left(C, R^{(p)}\right)$. Indeed,

$$
\begin{aligned}
{[\alpha, f](c) } & =(-1)^{\left|c^{\prime}\right||f|} \alpha\left(c^{\prime}\right) f\left(c^{\prime \prime}\right)-(-1)^{|f||\alpha|+|\alpha|\left|c^{\prime}\right|} f\left(c^{\prime}\right) \alpha\left(c^{\prime \prime}\right) \\
& =(-1)^{\left|c^{\prime}\right||f|} \alpha\left(c^{\prime}\right) f\left(c^{\prime \prime}\right)-(-1)^{|f||\alpha|+|\alpha|\left|c^{\prime \prime}\right|+\left|c^{\prime}\right|\left|c^{\prime \prime}\right|} f\left(c^{\prime \prime}\right) \alpha\left(c^{\prime}\right) \\
& =(-1)^{\left|c^{\prime}\right||f|}\left[\alpha\left(c^{\prime}\right), f\left(c^{\prime \prime}\right)\right],
\end{aligned}
$$

where the second equality follows from the fact that $C$ is cocommutative. Since $\alpha\left(c^{\prime}\right) \in \mathcal{L}$ and $f\left(c^{\prime \prime}\right) \in R^{(p)}$, we have $\left[\alpha\left(c^{\prime}\right), f\left(c^{\prime \prime}\right)\right] \in R^{(p)}$, because the map (2.13) is a morphism of $\mathcal{L}$ modules (with the adjoint action). This shows that the isomorphism $(2.18)$ is a morphism of $\operatorname{graded} \operatorname{Hom}(C, \mathcal{L})$ Lie modules. In particular, for $f \in \operatorname{Hom}\left(C, R^{(p)}\right)$, we have $[\iota, f] \in \operatorname{Hom}\left(C, R^{(p)}\right)$. The differential on $\operatorname{Hom}^{\iota}(C, R)$ thus restricts to $\operatorname{Hom}^{\iota}\left(C, R^{(p)}\right)$ for each $p$. Hence, the isomorphism (2.18) is an isomorphism of complexes

$$
\operatorname{Hom}^{\iota}(C, R) \cong \bigoplus_{\substack{p=0 \\ \text { Cambridge University Press }}}^{\infty} \operatorname{Hom}^{\iota}\left(C, R^{(p)}\right)
$$


Next, the Jacobi identity for the commutator bracket on the convolution algebra $\operatorname{Hom}(C, R)$ implies that the action of $\operatorname{Hom}(C, \mathcal{L})$ on $\operatorname{Hom}(C, R)$ is compatible with the twisted differential $\partial+[\iota,-]$. This proves the first statement of the theorem. The second statement follows from Proposition 2.2 once we verify that

$$
\mathrm{H}_{-*}\left[\operatorname{Hom}^{\iota}\left(C, R^{(p)}\right)\right] \cong \mathrm{H}^{*}\left(\mathfrak{a}, \operatorname{Sym}^{p}(\mathfrak{a})\right) .
$$

Since $C$ is Koszul dual to $R, R \otimes_{\iota} C$ is a semifree resolution of $k$ as a DG left $R \cong \mathcal{U} \mathcal{L}$-module. Similarly, since $C$ is Koszul dual to $\mathfrak{a}, \mathcal{U} \mathfrak{a} \otimes_{\tau} C$ is a semifree resolution of $k$ as a DG left $\mathcal{U} \mathfrak{a}$-module, where $\tau$ denotes the composite twisting cochain $C \stackrel{\iota}{\rightarrow} \mathcal{L} \stackrel{\sim}{\rightarrow} \mathfrak{a}$. It follows that there are isomorphisms in the derived category of complexes of $k$-vector spaces:

$$
\begin{aligned}
\operatorname{Hom}^{\iota}\left(C, R^{(p)}\right) & \cong \operatorname{Hom}_{\mathcal{U L}}\left(R \otimes_{\iota} C, \operatorname{Sym}^{p}(\mathcal{L})\right) \\
& \cong \operatorname{RHom}_{\mathcal{L}}\left(k, \operatorname{Sym}^{p}(\mathcal{L})\right) \\
& \cong \operatorname{RHom}_{\mathcal{L L}}\left(k, \operatorname{Sym}^{p}(\mathfrak{a})\right) \\
& \cong \operatorname{Hom}_{\mathcal{L}}\left(R \otimes_{\iota} C, \operatorname{Sym}^{p}(\mathfrak{a})\right) \\
& \cong \operatorname{Hom}^{\tau}\left(C, \operatorname{Sym}^{p}(\mathfrak{a})\right) \\
& \cong \operatorname{Hom}_{\mathfrak{a} \mathfrak{a}}\left(\mathcal{U} \mathfrak{a} \otimes_{\tau} C, \operatorname{Sym}^{p}(\mathfrak{a})\right) \\
& \cong \operatorname{RHom}_{\mathfrak{a}}\left(k, \operatorname{Sym}^{p}(\mathfrak{a})\right) .
\end{aligned}
$$

This implies the isomorphism (2.19) on homologies.

Let $E:=C^{*}$ denote the (graded) linear dual of $C$. Then $\operatorname{Hom}(\bar{E}[1], E)$ is an $E$-module via the action $(x \cdot f)(y)=x \cdot f(y)$ for $f \in \operatorname{Hom}(\bar{E}[1], E), x \in E, y \in \bar{E}[1]$. It is easy to verify that

$$
\operatorname{Hom}\left(C, R^{(p)}\right) \cong \operatorname{Sym}_{E}^{p}\left[\mathcal{L}_{E}(\operatorname{Hom}(\bar{E}[1], E))\right],
$$

where $\mathcal{L}_{E}(V)$ denotes the free Lie algebra generated (over $E$ ) by a free $E$-module $V$. From Theorem 2.2 and Corollary 2.2, we have the following:

Corollary 2.3. There is a direct sum decomposition

$$
\mathrm{C}_{\oplus}^{*}(E, E) \cong \bigoplus_{p=0}^{\infty} \operatorname{Sym}_{E}^{p}\left[\mathcal{L}_{E}(\operatorname{Hom}(\bar{E}[1], E))\right]
$$

Moreover,

$$
\mathrm{H}^{*}\left(\operatorname{Sym}_{E}^{p}\left[\mathcal{L}_{E}(\operatorname{Hom}(\bar{E}[1], E))\right]\right) \cong \mathrm{H}^{*}\left(\mathfrak{a} ; \operatorname{Sym}^{p}(\mathfrak{a})\right)
$$

Remark. The decomposition of Hochschild cochains in Corollary 2.3 is analogous to the Hodge decomposition of the complex of polydifferential operators on a smooth proper variety (over a field of characteristic 0) in [54, Section 4].

\section{Cyclic pairings and derived Poisson structures}

The notion of a (noncommutative) derived Poisson algebra was introduced in [2] (see also [7]), as a natural - higher homological - generalisation of the $\mathrm{H}_{0}$-Poisson algebras of Crawley-Boevey [16]. The $\mathrm{H}_{0}$-Poisson algebras can be viewed, in turn, as a generalisation of the so-called necklace Lie algebras introduced in [8, 30] following an idea of Kontsevich [40]. 


\subsection{Derived Poisson algebras}

Let $A$ be an augmented DG algebra. The space $\operatorname{Der}(A)$ of graded $k$-linear derivations of $A$ is naturally a DG Lie algebra with respect to the commutator bracket. Let $\underline{\operatorname{Der}}(A)^{\natural}$ denote the subcomplex of $\operatorname{Der}(A)$ consisting of the derivations with image in $k+[A, A] \subseteq A$. It is easy to see that $\underline{\operatorname{Der}}(A)^{\natural}$ is a $\overline{\mathrm{DG}}$ Lie ideal of $\underline{\operatorname{Der}}(A)$, so that $\underline{\operatorname{Der}}(A)_{\natural}:=\underline{\operatorname{Der}}(A) / \underline{\operatorname{Der}}(A)^{\natural}$ is a DG Lie algebra. The natural action of $\underline{\operatorname{Der}}(A)$ on $A$ induces a Lie algebra action of $\underline{\operatorname{Der}}(A)_{\natural}$ on the quotient space $A_{\natural}:=A /(k+[A, A])$. We write $\varrho: \underline{\operatorname{Der}}(A)_{\natural} \rightarrow \underline{\operatorname{End}}\left(A_{\natural}\right)$ for the corresponding DG Lie algebra homomorphism.

Now, following [2], we define a Poisson structure on $A$ to be a DG Lie algebra structure on $A_{\natural}$ such that the adjoint representation ad : $A_{\natural} \rightarrow \underline{\text { End }}\left(A_{\natural}\right)$ factors through $\varrho-$ that is, there is a map of DG Lie algebras $\alpha: A_{\natural} \rightarrow \underline{\operatorname{Der}}(A)_{\natural}$ such that ad $=\varrho \circ \alpha$. It is easy to see that if $A$ is a commutative DG algebra, then a Poisson structure on $A$ is the same thing as a (graded) Poisson bracket on $A$. On the other hand, if $A$ is an ordinary $k$-algebra (viewed as a DG algebra), then a Poisson structure on $A$ is precisely an $\mathrm{H}_{0}$-Poisson structure in the sense of [16].

Let $A$ and $B$ be two Poisson DG algebras - that is, objects of $\mathrm{DGA}_{k / k}$ equipped with Poisson structures. A morphism $f: A \rightarrow B$ of Poisson algebras is then a morphism $f: A \rightarrow B$ in $\mathrm{DGA}_{k / k}$ such that $f_{\natural}: A_{\natural} \rightarrow B_{\natural}$ is a morphism of DG Lie algebras. With this notion of morphisms, the Poisson DG algebras form a category which we denote $\mathrm{DGPA}_{k}$. Note that $\mathrm{DGPA}_{k}$ comes with two natural functors: the forgetful functor $U: \mathrm{DGPA}_{k} \rightarrow \mathrm{DGA}_{k / k}$ and the cyclic functor $(-)_{4}: \mathrm{DGPA}_{k} \rightarrow \mathrm{DGLA}_{k}$. We say that a morphism $f$ is a weak equivalence in $\mathrm{DGPA}_{k}$ if $U f$ is a weak equivalence in $\mathrm{DGA}_{k / k}$ and $f_{\mathfrak{b}}$ is a weak equivalence in $\mathrm{DGLA}_{k}$; in other words, a weak equivalence in $\mathrm{DGPA}_{k}$ is a quasi-isomorphism of DG algebras, $f: A \rightarrow B$, such that the induced map $f_{\natural}: A_{\natural} \rightarrow B_{\natural}$ is a quasi-isomorphism of DG Lie algebras.

Proposition 3.1 ([7]). The category DGPA $_{k}$ with weak equivalences is a (saturated) homotopical category in the sense of [21].

This proposition allows us to define a well-behaved homotopy category of Poisson algebras

$$
\operatorname{Ho}\left(\operatorname{DGPA}_{k}\right):=\operatorname{DGPA}_{k}\left[\mathscr{W}^{-1}\right]
$$

where $\mathscr{W}$ is the class of weak equivalences.

Definition 3.1 ([7]). A derived Poisson algebra is a cofibrant associative DG algebra A equipped with a Poisson structure, which is viewed up to weak equivalence - that is, as an object in $\mathrm{Ho}\left(\mathrm{DGPA}_{k}\right)$.

An important result that motivates our study of these objects is the following theorem generalising the main theorem of [16].

Theorem 3.1 (see $[2,7])$. Let $A$ be a derived Poisson algebra over $k$.

(a) The (reduced) cyclic homology $\overline{\mathrm{HC}}_{*}(A)$ of A carries a natural structure of a graded Lie algebra.

(b) For any $n \geq 1$, there is a unique graded Poisson algebra structure on the $n$-dimensional representation homology $\operatorname{HR}_{*}\left(A, k^{n}\right)$ GL of $A$, such that the derived character map (compare $[4,5,6]$ )

$$
\operatorname{Tr}_{n}(A): \overline{\mathrm{HC}}_{*}(A) \rightarrow \operatorname{HR}_{*}\left(A, k^{n}\right)^{\mathrm{GL}}
$$

is a (graded) Lie algebra homomorphism.

Example 3.1 (Necklace Lie algebras). The simplest example of a derived Poisson algebra is the tensor algebra $A=T_{k} V$ generated by an even-dimensional $k$-vector space $V$ equipped with a symplectic form $\langle-,-\rangle: V \times V \rightarrow V$. In this case, $A$ carries a double Poisson structure in the sense of [59]. The double bracket

$$
\{\{-,-\}\}: \bar{A} \otimes \bar{A} \rightarrow A \otimes A
$$


is given by the formula

$$
\begin{aligned}
& \left\{\left\{\left(v_{1}, \ldots, v_{n}\right),\left(w_{1}, \ldots, w_{m}\right)\right\}\right\}= \\
& \sum_{\substack{i=1, \ldots, n \\
j=1, \ldots, m}}\left\langle v_{i}, w_{j}\right\rangle\left(w_{1}, \ldots, w_{j-1}, v_{i+1}, \ldots, v_{n}\right) \otimes\left(v_{1}, \ldots, v_{i-1}, w_{j+1}, \ldots, w_{m}\right),
\end{aligned}
$$

where $\left(v_{1}, \ldots, v_{n}\right)$ denotes the element $v_{1} \otimes \cdots \otimes v_{n} \in T_{k} V$ with $v_{1}, \ldots, v_{n} \in V$. This double bracket can be extended to $A \otimes A$ by setting $\{\{a, 1\}\}=\{\{1, a\}\}=0$. It induces a noncommutative Poisson structure on $A$ with Lie bracket on $A_{\natural}$ given by

$$
\{\bar{\alpha}, \bar{\beta}\}=\overline{\mu \circ\{\{\alpha, \beta\}\}}
$$

where $\mu: A \otimes A \rightarrow A$ is the multiplication map and $\bar{a}$ denotes the image of $a \in A$ under the canonical projection $A \rightarrow A_{\natural}$. The Lie algebra $A_{\natural}=T_{k} V_{\natural}$ with this bracket is called the necklace Lie algebra $[8,30]$.

\subsection{Cyclic pairings}

We now describe a construction of derived Poisson structures associated with cyclic coalgebras. Recall (compare [27]) that a graded associative $k$-algebra is called $n$-cyclic if it carries a symmetric bilinear pairing $\langle-,-\rangle: A \times A \rightarrow k$ of degree $n$ satisfying

$$
\langle a b, c\rangle=\langle a, b c\rangle, \quad \forall a, b, c \in A .
$$

Dually, a graded coalgebra $C$ is called $n$-cyclic if it carries a symmetric bilinear pairing $\langle-,-\rangle: C \times C \rightarrow k$ of degree $n$ satisfying

$$
\left\langle v^{\prime}, w\right\rangle v^{\prime \prime}= \pm\left\langle v, w^{\prime \prime}\right\rangle w^{\prime}, \quad \forall v, w \in C,
$$

where $v^{\prime}$ and $v^{\prime \prime}$ are the two components of the coproduct $\Delta_{C} v=v^{\prime} \otimes v^{\prime \prime}$ written in Sweedler notation. Note that if $A$ is a finite-dimensional graded $-n$-cyclic algebra whose cyclic pairing is nondegenerate, then $C:=\operatorname{Hom}_{k}(A, k)$ is a graded $n$-cyclic coalgebra. A DG coalgebra $C$ is $n$-cyclic if it is $n$-cyclic as a graded coalgebra and

$$
\langle d u, v\rangle \pm\langle u, d v\rangle=0
$$

for all homogeneous $u, v \in C$ - that is, if $\langle-,-\rangle: C[n] \otimes C[n] \rightarrow k[n]$ is a map of complexes. We say that a coaugmented DG coalgebra $C \in \mathrm{DGC}_{k / k}$ is $n$-cyclic if $\bar{C}$ is $n$-cyclic as a noncounital DG coalgebra.

Assume that $C \in \mathrm{DGC}_{k / k}$ is equipped with a cyclic pairing of degree $n$ and let $R:=\mathbf{\Omega}(C)$ denote the (associative) cobar construction of $C$. Recall that $R=T_{k}(\bar{C}[-1])$ as a graded $k$-algebra. For $v_{1}, \ldots, v_{n} \in \bar{C}[-1]$, let $\left(v_{1}, \ldots, v_{n}\right)$ denote the element $v_{1} \otimes \cdots \otimes v_{n}$ of $R$. By [2, Theorem 15], the cyclic pairing on $C$ of degree $n$ induces a double Poisson bracket of degree $n+2$ (in the sense of [59])

$$
\{\{-,-\}\}: \bar{R} \otimes \bar{R} \rightarrow R \otimes R .
$$

This double bracket is given by the formula

$$
\begin{aligned}
& \left\{\left\{\left(v_{1}, \ldots, v_{n}\right),\left(w_{1}, \ldots, w_{m}\right)\right\}\right\}= \\
& \sum_{\substack{i=1, \ldots, n \\
j=1, \ldots, m}} \pm\left\langle v_{i}, w_{j}\right\rangle\left(w_{1}, \ldots, w_{j-1}, v_{i+1}, \ldots, v_{n}\right) \otimes\left(v_{1}, \ldots, v_{i-1}, w_{j+1}, \ldots, w_{m}\right)
\end{aligned}
$$


generalising equation (3.1). This double bracket can be extended to $R \otimes R$ by setting $\{\{r, 1\}\}=$ $\{\{1, r\}\}=0$. Associated to equation (3.2) is the usual bracket

$$
\{-,-\}:=\mu \circ\{\{-,-\}\}: R \otimes R \rightarrow R .
$$

Similarly, the bimodule $R \otimes R$ (equipped with outer $R$-bimodule structure) has a double bracket (in the sense of [15, Definition 3.5]) given by the formula

$$
\begin{aligned}
& \{\{-,-\}\}: R \times(R \otimes R) \rightarrow R \otimes(R \otimes R) \oplus(R \otimes R) \otimes R \\
& \{\{r, p \otimes q\}\}:=\{\{r, p\}\} \otimes q+(-1)^{|p|(|r|+n)} p \otimes\{\{r, q\}\} .
\end{aligned}
$$

This double bracket restricts to a double bracket on the sub-bimodule $\Omega^{1} R$ of $R \otimes R$ [15, Corollary 5.2]. Let $\{-,-\}: R \otimes \Omega^{1} R \rightarrow \Omega^{1} R$ denote the composite map $\mu \circ\{\{-,-\}\}$, where $\mu$ is the bimodule action. Furthermore, for any $R$-bimodule $M$, denote by $M_{\natural}:=M /[M, R]$ its abelianisation. Then for $M=\Omega^{1} R$, define $\{-,-\}_{\natural}: R \otimes \Omega^{1} R \rightarrow\left(\Omega^{1} R\right)_{\natural}$ to be the composition of $\{-,-\}$ with canonical projection দ: $\Omega^{1} R \rightarrow\left(\Omega^{1} R\right)_{\text {七. }}$.

As in the case of necklace Lie algebras, the bracket $\nvdash \circ\{-,-\}: R \otimes R \rightarrow R_{\natural}$ descends to a DG $(n+2)$ Poisson structure on $R$. In particular, there is a (DG) Lie bracket $\{-,-\}_{\natural}$ on $R_{\natural}$ of degree $n+2$. The restriction of the bracket (3.3) to $\bar{R}$ induces a degree $n+2$ DG Lie module structure over $R_{\natural}$ on $\bar{R}$, and the bracket $\{-,-\}_{\natural}: R \otimes \Omega^{1} R \rightarrow\left(\Omega^{1} R\right)_{\natural}$ induces a degree $n+2$ DG Lie module structure over $R_{\natural}$ on $\left(\Omega^{1} R\right)_{\natural}$ [15, Proposition 3.11]. On homologies, we have the following (see [15, Theorems 1.1 and 1.2]):

Theorem 3.2. Let $A \in \mathrm{DGA}_{k / k}$ be an augmented $D G$ algebra Koszul dual to a DG coalgebra $C \in$ $\mathrm{DGC}_{k / k}$. Assume that $C$ is $n$-cyclic. Then $\overline{\mathrm{HC}}_{*}(A)$ has the structure of a graded Lie algebra with Lie bracket of degree $n+2$. Moreover, $\overline{\mathrm{HH}}_{*}(A)$ has the structure of a graded Lie module over $\overline{\mathrm{HC}}_{*}(A)$ of degree $n+2$, with Connes' maps I, B and $S$ being the degree $(n+2)$ Lie module homomorphisms over $\overline{\mathrm{HC}}_{*}(A)$.

The Lie bracket of degree $n+2$ on $\overline{\mathrm{HC}}_{*}(A)$ induced by an $(n+2)$-Poisson structure on $R_{\natural}$ as before is an example of a derived $(n+2)$-Poisson structure on $A$. Such derived Poisson structures have been further studied in $[7,55]$.

\section{Convention}

Since we work with algebras that are Koszul dual to $n$-cyclic coalgebras, the associated Lie algebras that we work with have Lie bracket of degree $n+2$. Similarly, the modules over such Lie algebras are Lie modules of degree $n+2$. To simplify our terminology, we will drop the prefix 'degree $n+2$ ' in all sections that follow. In particular, for $n$ fixed, we will refer to (derived) $(n+2)$-Poisson structures simply as (derived) Poisson structures.

\subsection{Van den Bergh duality}

Assume that $A \in \mathrm{DGA}_{k / k}$ is Koszul dual to $C \in \mathrm{DGC}_{k / k}$. Let $\tau: C \rightarrow A$ denote the twisting cochain corresponding to the quasi-isomorphism $R \stackrel{\sim}{\rightarrow} A$, where $R:=\mathbf{\Omega}(C)$. Further, assume that $C$ is a finite-dimensional coalgebra equipped with a cyclic pairing (of degree $-n$ ) which is induced by a nondegenerate cyclic pairing (of degree $n$ ) on the graded linear dual $E=C^{*}$. The pairing on $C$ induces an isomorphism (complexes) $\phi: E:=\operatorname{Hom}_{k}(C, k) \rightarrow C[-n]$ of $k$-vector spaces whose (shifted) inverse is the linear map

$$
C \cong E[n], \quad c \mapsto\langle c,-\rangle .
$$


The isomorphism $\phi: E \rightarrow C[-n]$ induces an isomorphism of DG $R$-bimodules

$$
\operatorname{Hom}_{k}^{\iota}\left(C, R^{e}\right) \rightarrow R \otimes_{\iota} C[-n] \otimes_{\iota} R,
$$

where the $R$-bimodule structure on the left is induced by the 'inner' bimodule structure on $R^{e}$. Identifying $\operatorname{Hom}^{\iota}\left(C, R^{e}\right)=\operatorname{Hom}_{R^{e}}\left(R \otimes_{\iota} C \otimes_{\iota} R, R^{e}\right)$ and noting that $R \otimes_{\iota} C \otimes_{\iota} R$ is a semifree resolution of $R$, we see that the nondegenerate cyclic pairing on $C$ induces an isomorphism in $\mathcal{D}^{b}\left(R^{e}\right)$

$$
R^{\vee} \cong R[-n],
$$

where $R^{\vee}$ is the (derived) bimodule dual of $R$. Taking derived tensor products over $R^{e}$ and homology, we obtain an isomorphism

$$
\Psi: \mathrm{HH}^{*}(A, A) \cong \mathrm{HH}_{n-*}(A) .
$$

The isomorphism $R^{\vee} \cong R[-n]$ induces an isomorphism $\mathrm{H}_{*}\left(R^{\vee} \otimes_{R^{e}}^{\boldsymbol{L}} R\right) \cong \mathrm{H}_{*}\left(R \otimes_{R^{e}}^{\boldsymbol{L}} R[-n]\right)$. The image of the identity map on $R$ (viewed as an element of $\mathrm{H}_{0}\left(R^{\vee} \otimes_{R^{e}}^{\boldsymbol{L}} R\right)$ under this isomorphism) is an element $\eta \in \mathrm{HH}_{n}(A, A)$. We recall the following:

Lemma 3.1 ([17, Proposition 5.5]). The map $\Psi$ coincides with the isomorphism $\eta \cap-: \mathrm{HH}^{*}(A, A) \cong$ $\mathrm{HH}_{n-*}(A)$.

Let $\{-,-\}: \overline{\mathrm{HC}}_{*}(A) \otimes \overline{\mathrm{HC}}_{*}(A) \rightarrow \overline{\mathrm{HC}}_{*}(A)$ denote the derived Poisson bracket on $\overline{\mathrm{HC}}_{*}(A)$. The following result was proven for quadratic Koszul algebras in [15, proof of Corollary 1.5]; we give a different, more direct proof in a slightly more general context:

Proposition 3.2. The derived Poisson bracket on $\overline{\mathrm{HC}}_{*}(A)$ is given by

$$
\{\alpha, \beta\}=I\left[\Psi\left[\Psi^{-1}(B(\alpha)) \cup \Psi^{-1}(B(\beta))\right]\right], \quad \forall \alpha, \beta \in \overline{\mathrm{HC}}_{*}(A) .
$$

Proof. Since $R \otimes_{\iota} C \otimes_{\iota} R$ is a semifree resolution of $R$ as an $R$-bimodule, $\mathrm{HH}_{*}(A) \cong \mathrm{HH}_{*}(R)$ can be identified with the homology of the complex $\left(R \otimes_{\iota} C \otimes_{\iota} R\right) \otimes_{R^{e}} R$, which is isomorphic to $R \otimes C$ as graded vector spaces. The differential on $R \otimes C$ induced by that on $R \otimes_{\iota} C \otimes_{\iota} R$ is, however, twisted and differs from the differential on $R \otimes_{\iota} C$. We let $R \otimes_{\iota} C_{\iota}$ denote $R \otimes C$ equipped with this differential. Explicitly, for $r \in R$ and $c \in C$, we have

$$
\partial_{R \otimes_{\iota} C_{\iota}}(r \otimes c)=d_{R} r \otimes c+(-1)^{|r|} r \otimes d_{C} c+(-1)^{\left|c^{\prime \prime}\right|\left(|r|+\left|c^{\prime}\right|\right)} \tau\left(c^{\prime \prime}\right) r \otimes c^{\prime}-(-1)^{|r|} r \tau\left(c^{\prime}\right) \otimes c^{\prime \prime} .
$$

On the other hand, by a theorem of Feigin and Tsygan [22] (see also [5]), $\overline{\mathrm{HC}}_{*}(A) \cong \mathrm{H}_{*}\left[R_{\natural}\right]$. It is easy to verify that the Lie bracket on $R_{\natural}$ is given by the composite map

$$
R_{\natural} \otimes R_{\natural} \stackrel{\partial \otimes \partial}{\longrightarrow}\left(R \otimes_{\iota} C_{\iota}[-1]\right) \otimes\left(R \otimes_{\iota} C_{\iota}[-1]\right) \rightarrow R \otimes R \otimes C[-1]^{\otimes 2} \stackrel{\mu \otimes\langle-,-\rangle}{\longrightarrow} R \rightarrow R_{\natural},
$$

where the second arrow permutes factors and $\partial: R_{\natural} \rightarrow R \otimes_{\iota} C_{\iota}[-1]$ denotes the cyclic derivative. On homology, the cyclic derivative $\partial$ induces the Connes operator $B: \overline{\mathrm{HC}}_{*}(A) \rightarrow \mathrm{HH}_{*+1}(A)$. It therefore suffices to check that the map induced on homology by the composition

$$
\left(R \otimes_{\iota} C_{\iota}\right) \otimes\left(R \otimes_{\iota} C_{\iota}\right) \rightarrow R \otimes R \otimes C^{\otimes 2} \stackrel{\mu \otimes\langle-,-\rangle}{\longrightarrow} R \rightarrow R_{\natural}
$$

coincides with

$$
\mathrm{HH}_{*}(A)^{\otimes 2} \stackrel{\left(\Psi^{-1}\right)^{\otimes 2}}{\rightarrow} \mathrm{HH}^{*}(A, A) \stackrel{\otimes 2}{\rightarrow} \stackrel{\cup}{\rightarrow} \mathrm{HH}^{*}(A, A) \stackrel{\Psi}{\rightarrow} \mathrm{HH}_{*}(A) \stackrel{\mathrm{I}}{\rightarrow} \overline{\mathrm{HC}}_{*}(A) .
$$

By Proposition 2.2, $\mathrm{HH}^{*}(A, A)$ is the homology of $\operatorname{Hom}^{\iota}(C, R)$, whose convolution product induces the cup product. On identifying $\operatorname{Hom}^{\iota}(C, R)$ with $R \otimes E$ as graded vector spaces, the map $\Psi^{-1}$ gets 
identified with the map induced on homology by $\operatorname{Id}_{R} \otimes \phi^{-1}: R \otimes C \rightarrow R \otimes E$, and the convolution product on $\operatorname{Hom}^{\iota}(C, R)$ is identified with the product on $R \otimes E$. On the other hand, the map I is induced on homology by

$$
R \otimes_{\iota} C_{\iota} \stackrel{\mathrm{Id}_{R} \otimes \varepsilon_{C}}{\longrightarrow} R \rightarrow R_{\natural}
$$

It therefore suffices to verify that the following diagram commutes:

$$
\begin{gathered}
(R \otimes C) \otimes(R \otimes C) \longrightarrow(R \otimes R) \otimes(C \otimes C) \stackrel{\mu_{R} \otimes\langle-,-\rangle}{\longrightarrow} R \stackrel{\mathrm{Id}}{\longrightarrow} R . \\
\left(\operatorname{Id}_{R} \otimes \phi^{-1}\right)^{\otimes 2} \downarrow \quad \operatorname{Id}_{R^{\otimes 2}} \otimes\left(\phi^{-1}\right)^{\otimes 2} \downarrow \\
(R \otimes E) \otimes(R \otimes E) \longrightarrow(R \otimes R) \otimes(E \otimes E) \stackrel{\mu_{R} \otimes \mu_{E}}{\longrightarrow} R \otimes E \stackrel{\operatorname{Id}_{R} \otimes \varepsilon_{C}}{\longrightarrow} \stackrel{\operatorname{Id}_{R} \otimes \phi}{\longrightarrow} R \otimes C
\end{gathered}
$$

This reduces to verifying the commutativity of the diagram

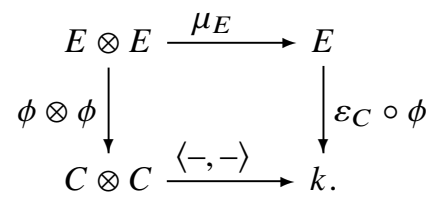

Note that $\varepsilon_{C}: C \rightarrow k$ coincides with $1_{E}$ under the identification $E=C^{*}$. Thus, for $v \in E$,

$$
\varepsilon_{C}(\phi(v))=1_{E}(\phi(v))=\left\langle\phi\left(1_{E}\right), \phi(v)\right\rangle_{C}=\left\langle 1_{E}, v\right\rangle_{E},
$$

where $\langle-,-\rangle_{E}$ denotes the original pairing on $E$. The commutativity of the diagram therefore follows once we show that

$$
\langle\phi(v), \phi(w)\rangle=\langle v, w\rangle_{E}=\left\langle 1_{E}, v \cdot w\right\rangle_{E}, \quad \forall v, w \in E .
$$

This is a consequence of the fact that the pairing on $E$ is cyclic. This completes the proof.

Recall (see Theorem 3.2) that there is an action of $\overline{\mathrm{HC}}_{*}(A)$ on $\mathrm{HH}_{*}(A)$ making the latter a graded Lie module over the former. Abusing notation, we denote this action by

$$
\{-,-\}: \overline{\mathrm{HC}}_{*}(A) \times \mathrm{HH}_{*}(A) \rightarrow \mathrm{HH}_{*}(A) .
$$

Let $[-,-]_{G}$ denote the Gerstenhaber bracket on $\operatorname{HH}^{*}(A, A)$.

Proposition 3.3. For all $\alpha \in \overline{\mathrm{HC}}_{*}(A)$ and all $\beta \in \mathrm{HH}_{*}(A)$,

$$
\{\alpha, \beta\}=\Psi\left(\left[\Psi^{-1}(B(\alpha)), \Psi^{-1}(\beta)\right]_{G}\right) .
$$

Proof. Note that the isomorphism $\Psi$ can be used to transport the Gerstenhaber bracket onto $\mathrm{HH}_{*}(A)$, making $\mathrm{HH}_{*}(A)$ a graded Lie algebra (up to shift in homological degree) with Lie bracket $\Psi\left(\left[\Psi^{-1}(-), \Psi^{-1}(-)\right]_{G}\right)$. By [15, Corollary 8.6 and proof of Theorem 1.6], $B: \overline{\mathrm{HC}}_{*}(A) \rightarrow \mathrm{HH}_{*+1}(A)$ is a graded Lie algebra homomorphism, where $\overline{\mathrm{HC}}_{*}(A)$ is equipped with the derived Poisson bracket. It therefore remains to verify that the action of $\overline{\mathrm{HC}}_{*}(A)$ on $\mathrm{HH}_{*}(A)$ arising out of the Lie algebra homomorphism $B$ coincides with the action arising out of the derived Poisson structure. We complete this verification in the routine computation that follows. For notational brevity, define $V:=\bar{C}[-1]$, and for $v_{1}, \ldots, v_{n} \in V$, set $\left(v_{1}, \ldots, v_{n}\right):=v_{1} \otimes \cdots \otimes v_{n} \in R$. Pick $p=\left(v_{1}, \ldots, v_{n}\right) \in R$ and $q \otimes c=\left(u_{1}, \ldots, u_{m}\right) \otimes c \in R \otimes_{\iota} C_{\iota}$. Then

$$
\{p, q \otimes c\}=\{p, q\} \otimes c+(-1)^{(|p|-n)|q|} \boxminus(q d\{p, c\}),
$$


where $\natural: \Omega^{1} R \rightarrow \Omega^{1} R_{\natural}$ denotes the canonical projection. Hence $\{p, q \otimes c\}$ equals

$$
\begin{aligned}
& \sum_{\substack{1 \leqslant i \leqslant n \\
1 \leqslant k \leqslant m}} \pm\left\langle s v_{i}, s u_{k}\right\rangle\left(u_{1}, \ldots, u_{k-1}, v_{i+1}, \ldots, v_{n}, v_{1}, \ldots, v_{i-1}, u_{k+1}, \ldots, u_{m}\right) \otimes c+ \\
& \sum_{1 \leqslant j \leqslant n} \pm G\left[\left(u_{1}, \ldots, u_{m}\right) d\left(\left\langle s v_{j}, c\right\rangle v_{j+1}, \ldots, v_{n}, v_{1}, \ldots, v_{j-1}\right)\right],
\end{aligned}
$$

or more explicitly,

$$
\begin{gathered}
\sum_{\substack{1 \leqslant i \leqslant n \\
1 \leqslant k \leqslant m}} \pm\left\langle s v_{i}, s u_{k}\right\rangle\left(u_{1}, \ldots, u_{k-1}, v_{i+1}, \ldots, v_{n}, v_{1}, \ldots, v_{i-1}, u_{k+1}, \ldots, u_{m}\right) \otimes c+ \\
\sum_{1 \leqslant i<j \leqslant n} \pm\left\langle s v_{j}, c\right\rangle\left(v_{i+1}, \ldots, v_{j-1}, u_{1}, \ldots, u_{m}, v_{j+1}, \ldots, v_{n}, v_{1}, \ldots, v_{i-1}\right) \otimes s v_{i}+ \\
\sum_{1 \leqslant j<i \leqslant n} \pm\left\langle s v_{j}, c\right\rangle\left(v_{i+1}, \ldots, v_{n}, v_{1}, \ldots, v_{j-1}, u_{1}, \ldots, u_{m}, v_{j+1}, \ldots, v_{i-1}\right) \otimes s v_{i}
\end{gathered}
$$

On the other hand,

$$
\begin{aligned}
\Psi^{-1}\left(B\left(v_{1}, \ldots, v_{n}\right)\right) & =\sum_{1 \leq i \leq n}\left(v_{i+1}, \ldots, v_{n}, v_{1}, \ldots, v_{i-1}\right) \otimes \widetilde{s v_{i}}, \\
\Psi^{-1}\left(\left(u_{1}, \ldots, u_{m}\right) \otimes c\right) & =\left(u_{1}, \ldots, u_{m}\right) \otimes \widetilde{c}
\end{aligned}
$$

where we denote $\widetilde{c}:=\phi^{-1}(c)$. Hence the bracket $\left[\Psi^{-1}(B(p)), \Psi^{-1}(q)\right]_{G}$ equals

$$
\begin{aligned}
& \sum_{1 \leqslant i<j \leqslant n} \pm s v_{j}(\widetilde{c})\left(v_{i+1}, \ldots, v_{j-1}, u_{1}, \ldots, u_{m}, v_{j+1}, \ldots, v_{n}, v_{1}, \ldots, v_{i-1}\right) \otimes \widetilde{s v_{i}}+ \\
& \sum_{1 \leqslant j<i \leqslant n} \pm s v_{j}(\widetilde{c})\left(v_{i+1}, \ldots, v_{n}, v_{1}, \ldots, v_{j-1}, u_{1}, \ldots, u_{m}, v_{j+1}, \ldots, v_{i-1}\right) \otimes \widetilde{s v_{i}}+ \\
& \sum_{\substack{1 \leqslant i \leqslant n \\
1 \leqslant k \leqslant m}} \pm s v_{i}\left(\widetilde{s u_{k}}\right)\left(u_{1}, \ldots, u_{k-1}, v_{i+1}, \ldots, v_{n}, v_{1}, \ldots, v_{i-1}, u_{k+1}, \ldots, u_{m}\right) \otimes \widetilde{c}
\end{aligned}
$$

Since $u(\widetilde{w})=\langle u, w\rangle$ for all $u, w \in C$, this computation shows that

$$
\Psi^{-1}(\{p, q \otimes c\})=\left[\Psi^{-1}(B(p)), \Psi^{-1}(q \otimes c)\right]_{G} .
$$

Since $\overline{\mathrm{HC}}_{*}(A)=\mathrm{H}_{*}\left(R_{\natural}\right)$ and $\mathrm{HH}_{*}(A)=\mathrm{H}_{*}\left(R \otimes_{\iota} C_{\iota}\right)$, the desired verification is complete once we apply $\Psi$ to both sides of this equation.

Assume further that $C$ is cocommutative, so that $A \cong \mathcal{U} \mathfrak{a}$, where $\mathfrak{a} \in \mathrm{DGLA}_{k}$ is Koszul dual to $C$. The image of the counit $\varepsilon_{C} \in C^{\vee}$ under the isomorphism $C^{\vee} \cong C[-n]$ defines an $n$-cycle in $C$ whose class in $\mathrm{H}_{n}(C) \cong \mathrm{H}_{n}(\mathfrak{a} ; k)$ is denoted by $\eta$. This in turn defines a cap product $\eta \cap-: \mathrm{H}^{n-r}(\mathfrak{a} ; N) \rightarrow \mathrm{H}_{r}(\mathfrak{a} ; N)$ for any DG a-module $N$ [25, Sect. 7.1].

Lemma 3.2. Under the natural isomorphisms $\mathrm{HH}_{*}(\mathcal{U} \mathfrak{a}) \cong \mathrm{H}_{*}\left(\mathfrak{a} ; \mathcal{U}_{\mathfrak{a}}\right)$ and $\mathrm{HH}^{*}\left(\mathcal{U}_{\mathfrak{a}}, \mathcal{U}_{\mathfrak{a}}\right) \cong \mathrm{H}^{*}\left(\mathfrak{a} ; \mathcal{U}_{\mathfrak{a}}\right)$, the map $\Psi$ is identified with

$$
\eta \cap-: \mathrm{H}^{*}\left(\mathfrak{a} ; \mathcal{U}_{\mathfrak{a}}\right) \rightarrow \mathrm{H}_{n-*}(\mathfrak{a} ; \mathcal{U} \mathfrak{a})
$$

As a consequence,

$$
\Psi\left[\mathrm{H}^{*}\left(\mathfrak{a} ; \operatorname{Sym}^{p}(\mathfrak{a})\right)\right]=\mathrm{H}_{n-*}\left(\mathfrak{a} ; \operatorname{Sym}^{p}(\mathfrak{a})\right), \quad \forall p \geq 0 .
$$


Proof. The map $\Psi$ is induced by the map of complexes

$$
\operatorname{Hom}^{\iota}(C, R) \cong R \otimes_{\iota} C_{\iota}[-n]
$$

obtained by tensoring the bimodule map (3.4) with $R$ over $R^{e}$. Hence, identifying the left-hand side (as graded vector spaces) with $R \otimes E$, we see that the isomorphism (3.5) coincides with the map $\operatorname{Id}_{R} \otimes \phi$.

On the other hand, the natural isomorphisms $\mathrm{HH}_{*}(\mathcal{U} \mathfrak{a}) \cong \mathrm{H}_{*}(\mathfrak{a} ; \mathcal{U} \mathfrak{a})$ and $\mathrm{HH}^{*}(\mathcal{U} \mathfrak{a}, \mathcal{U} \mathfrak{a}) \cong \mathrm{H}^{*}(\mathfrak{a} ; \mathcal{U} \mathfrak{a})$ are induced by the maps $\operatorname{Hom}^{\iota}(C, R) \rightarrow \operatorname{Hom}^{\tau}(C, \mathcal{U} \mathfrak{a})$ and $R \otimes_{\iota} C_{\iota} \rightarrow \mathcal{U} \mathfrak{a} \otimes_{\tau} C_{\tau}$ induced by the canonical projection $R \stackrel{\sim}{\rightarrow} \mathcal{U} \mathfrak{a}$, and where $\tau: C \rightarrow \mathcal{U} \mathfrak{a}$ denotes the twisting cochain corresponding to the algebra homomorphism $R \stackrel{\sim}{\rightarrow}$ Ua . We therefore need to verify that the map $\eta \cap-$ is induced on homologies by the map

$$
\operatorname{Id}_{\mathcal{U} \mathfrak{a}} \otimes \phi: \operatorname{Hom}^{\tau}(C, \mathcal{U} \mathfrak{a}) \rightarrow \mathcal{U}_{\mathfrak{a}} \otimes_{\tau} C_{\tau}[-n]
$$

Identifying $\operatorname{Hom}^{\tau}(C, \mathcal{U} \mathfrak{a})=\mathcal{U} \mathfrak{a} \otimes E$ as a graded vector space, we see that by [25, Section 7.1], the map $\eta \cap-$ is induced on homology by a map of complexes which coincides (as a map of graded vector spaces) with

$$
\mathcal{U}_{\mathfrak{a}} \otimes E \stackrel{\mathrm{Id}_{\mathcal{U} \otimes \otimes E} \otimes \eta}{\longrightarrow} \mathcal{U} \mathfrak{a} \otimes E \otimes C[-n] \stackrel{\mathrm{Id} \otimes \Delta}{\longrightarrow} \mathcal{U} \mathfrak{a} \otimes E \otimes C \otimes C[-n] \stackrel{\mathrm{Id}_{\mathcal{U}_{\mathfrak{a}} \otimes \mathrm{ev} \otimes \mathrm{Id}}^{\longrightarrow}}{\longrightarrow} \mathcal{U a}_{\mathfrak{a}} \otimes C[-n] .
$$

It therefore, suffices to check that $\Phi: E \rightarrow C[-n]$ coincides with the map

$$
E \stackrel{\mathrm{Id}_{E} \otimes \eta}{\longrightarrow} E \otimes C[-n] \stackrel{\mathrm{Id}_{E} \otimes \Delta}{\longrightarrow} E \otimes C \otimes C[-n] \stackrel{\mathrm{ev} \otimes \mathrm{Id}}{\longrightarrow} C[-n]
$$

which is clear. This completes the proof.

\section{Hodge decomposition of derived Poisson structures}

\subsection{The main theorem}

Recall that if $\mathfrak{g}$ is an $L_{\infty}$-algebra with higher operations (Lie brackets) $m_{k}: \wedge^{k} \mathfrak{g} \rightarrow \mathfrak{g}, k \geq 1$, the lower central filtration on $\mathfrak{g}$ is defined inductively (see, e.g., [26, Sect. 4]) by

$$
F^{1} \mathfrak{g}:=\mathfrak{g}, \quad F^{r} \mathfrak{g}:=\sum_{i_{1}+\cdots+i_{k}=r} m_{k}\left(F^{i_{1}} \mathfrak{g}, \ldots, F^{i_{k}} \mathfrak{g}\right), \quad r \geq 2
$$

Then $\mathfrak{g}$ is called nilpotent if definition (4.1) terminates after finitely many steps - that is, $F^{r} \mathfrak{g}=0$ for $r \gg 0$. For the rest of this section, we assume the following:

Assumption: $\mathfrak{g}$ is a nonnegatively graded, finite-dimensional, nilpotent $L_{\infty}$-algebra.

This assumption implies that all higher Lie brackets $m_{k}$ vanish for $k \gg 0$. The Chevalley-Eilenberg cochain algebra of $\mathfrak{g}$ is therefore of the form $\mathcal{A}=(\operatorname{Sym}(V), Q)$, where $V=\mathfrak{g}^{*}[-1]$. Let $W:=\mathfrak{g}[1]$ denote the (graded) linear dual of $V$. Following [13, Section 5], we will use the language of formal differential geometry, regarding $W$ as a supermanifold and $\mathcal{A}$ as the algebra of functions on $W$ equipped with cohomological vector field $Q$ of (cohomological) degree 1 . Note that the algebra $\operatorname{Sym}(V) \otimes \operatorname{Sym}(W[-1])$ of polyvector fields on $W$ is naturally a graded Lie module over the Lie algebra $\operatorname{Der}(\operatorname{Sym}(V)) \cong$ $\operatorname{Sym}(V) \otimes W$ of (graded) derivations of $\operatorname{Sym}(V)$. Let $\mathcal{V}$ denote the algebra of polyvector fields on $W$ equipped with the differential given by the action of the derivation $Q \in \operatorname{Der}(\operatorname{Sym}(V))$. The Schouten 
bracket makes $\mathcal{V}$ a Gerstenhaber algebra. Recall that there is a natural Hochschild-Kostant-Rosenberg map $\mathrm{I}_{\mathrm{HKR}}: \mathcal{V} \rightarrow \mathrm{C}_{\oplus}^{*}(\mathcal{A}, \mathcal{A})$, which is defined (on homogeneous derivations of $\mathcal{A}$ ) by the formula

$$
\mathrm{I}_{\mathrm{HKR}}\left(\partial_{1} \cdots \partial_{n}\right):=\frac{1}{n !} \sum_{\sigma \in \Sigma_{n}} \pm \operatorname{sign}(\sigma) \partial_{\sigma(1)} \otimes \cdots \otimes \partial_{\sigma(n)},
$$

where the extra signs come from the Koszul sign rule.

The next observation is crucial for the proof of our main theorem.

Proposition 4.1. The map $I_{\mathrm{HKR}}: \mathcal{V} \rightarrow \mathrm{C}_{\oplus}^{*}(\mathcal{A}, \mathcal{A})$ is a quasi-isomorphism of complexes that induces an isomorphism of algebras on cohomology.

Proof. While it is not hard to check directly that the map $\mathrm{I}_{\mathrm{HKR}}: \mathcal{V} \rightarrow \mathrm{C}_{\oplus}^{*}(\mathcal{A}, \mathcal{A})$ is a quasi-isomorphism of complexes, the fact that it induces an algebra isomorphism on cohomology is more subtle. To establish this fact we observe that $\mathrm{I}_{\mathrm{HKR}}$ takes values in the subcomplex $\mathrm{D}_{\text {poly }}^{*}(\mathcal{A})$ of $\mathrm{C}_{\oplus}^{*}(\mathcal{A}, \mathcal{A})$ spanned by multilinear maps $\mathcal{A}^{\otimes n} \rightarrow \mathcal{A}$ that are differential operators in each argument - in other words, the Hochschild-Kostant-Rosenberg map factors as

$$
\mathrm{I}_{\mathrm{HKR}}: \mathcal{V} \rightarrow \mathrm{D}_{\text {poly }}^{*}(\mathcal{A}) \hookrightarrow \mathrm{C}_{\oplus}^{*}(\mathcal{A}, \mathcal{A}) .
$$

We will prove that each of the two arrows in the composition (4.2) induces an algebra isomorphism on cohomology. For the second arrow, this follows from Lemma 4.1, which shows that the inclusion $\mathrm{D}_{\text {poly }}^{*}(\mathcal{A}) \hookrightarrow \mathrm{C}_{\oplus}^{*}(\mathcal{A}, \mathcal{A})$ is actually a quasi-isomorphism of DG algebras. As for the first arrow, we will use a general version of the Duflo-Kontsevich isomorphismtTheorem for symmetric algebras equipped with a (co)homological differential (see, e.g., [13, Theorem 5.3]). This last theorem implies that the map $\mathrm{I}_{\mathrm{HKR}}: \mathcal{V} \rightarrow \mathrm{D}_{\text {poly }}^{*}(\mathcal{A})$ induces an algebra isomorphism on cohomology, up to a twist given by a canonical cohomology class $J(\alpha)$ called the Todd genus. It suffices to show that under our assumptions on $\mathfrak{g}$, the class $J(\alpha)$ is trivial.

Recall that the graded algebra of differential forms on $W$ is defined by $\Omega(W):=\operatorname{Sym}(V \oplus V[1])$. For any $x \in V$ we write $d x$ for the corresponding element in $V$ [1]. The de Rham differential is the derivation of (homological) degree 1 given on generators by $d(x)=d x, d(d x)=0$. There is an action $\iota$ of differential forms on polyvector fields by contraction, where $x \in V$ acts by left multiplication and $d x$ acts by the derivation $\iota_{d x}$ such that $\iota_{d x}(y)=0$ for $y \in V$ and $\iota_{d x}\left(s^{-1} v\right)=x(v)$ for $v \in W$. Choosing a basis $\left\{x^{1}, \ldots, x^{n}\right\}$ in $V$ that consists of homogeneous elements, we define the 1 -form $\alpha \in \Omega^{1}(W) \otimes \operatorname{End}(W)$ whose matrix with respect to the basis $\left\{e_{i}:=\partial_{x^{i}}\right\}$ of $W$ is given by

$$
\alpha_{i}^{j}:=d\left(\partial_{x^{i}} Q\left(x^{j}\right)\right)=\frac{\partial^{2} Q\left(x^{j}\right)}{\partial x^{i} \partial x^{k}} d x^{k} .
$$

Let $\widehat{\Omega}(W)$ denote the completion of $\Omega(W)$ with respect to the ideal generated by $V[1]$. The Todd genus associated to $\alpha$ is defined by

$$
J(\alpha):=\operatorname{Ber}\left[\frac{1-\exp (-\alpha)}{\alpha}\right] \in \widehat{\Omega}(W),
$$

where Ber : $\widehat{\Omega}(W) \otimes \operatorname{End}(W) \rightarrow \widehat{\Omega}(W)$ is a map induced by the Berezinian on $\operatorname{End}(W)$ [43, Chap. I, §7]). Note that $\frac{1-\exp (-\alpha)}{\alpha}=\exp \left(\sum_{k} c_{k} \alpha^{k}\right)$ for some formal power series $\sum_{k} c_{k} \alpha^{k}$ with constant term 0 . Thus

$$
J(\alpha)=\exp \left(\sum_{k=1}^{\infty} c_{k} \operatorname{Str}\left(\alpha^{k}\right)\right),
$$

where $\operatorname{Str}: \Omega(W) \otimes \operatorname{End}(W) \rightarrow \Omega(W)$ is a linear map induced by the (super)trace on $\operatorname{End}(W)$. 
We claim that $J(\alpha)=1$. It suffices to show that $\alpha$ is nilpotent, since then $\operatorname{Str}\left(\alpha^{k}\right)=0$ for all $k$. The nilpotency of $\alpha$ follows from the nilpotency of the $L_{\infty}$-algebra $\mathfrak{g}=W[-1]$. Recall that the (co)restriction of the Chevalley-Eilenberg (co)derivation on $\mathcal{C}^{*}(\mathfrak{g} ; k)$ to $W=\mathfrak{g}[1]$ gives a linear map $\operatorname{Sym}(W) \rightarrow W$, whose component in degree $p$ we denote by

$$
[-, \ldots,-]_{p}: \operatorname{Sym}^{p}(W) \rightarrow W, \quad v_{1} \cdots v_{p} \mapsto\left[v_{1}, \ldots, v_{p}\right]_{p} .
$$

Note that $\left[v_{1}, \ldots, v_{p}\right]_{p}= \pm m_{p}\left(s^{-1} v_{1}, \ldots, s^{-1} v_{p}\right)$ for $v_{1}, \ldots, v_{p}$ homogeneous in $W$, where $m_{p}$ : $\mathfrak{g}^{\otimes p} \rightarrow \mathfrak{g}$ is the $p$-fold bracket on $\mathfrak{g}$ (which of course is antisymmetric). Now,

$$
Q\left(x^{j}\right)=-\sum_{p \geqslant 1} \sum_{i_{1}, \ldots, i_{p}} \frac{1}{p !}\left[e_{i_{1}}, \ldots, e_{i_{p}}\right]_{p}^{j} x^{i_{1}} \cdots x^{i_{p}},
$$

where $v^{j}$ stands for the coefficient of $e_{j}$ in $V$ for any $v \in W$. Therefore

$$
\begin{aligned}
\alpha\left(e_{i}\right) & =\partial_{x_{i}} d Q\left(x^{j}\right) e_{j}=-\sum_{p \geqslant 2} \sum_{i_{1}, \ldots, i_{p}} \frac{1}{(p-2) !}\left[e_{i_{1}}, e_{i_{2}}, \ldots, e_{i_{p}}\right]_{p} d x^{i_{1}} \delta_{i i_{2}} x^{i_{3}} \cdots x^{i_{p}} \\
& =-\sum_{p \geqslant 2} \sum_{i_{1}, i_{3}, \ldots, i_{p}} \frac{1}{(p-2) !}\left[e_{i_{1}}, e_{i}, \ldots, e_{i_{p}}\right]_{p} d x^{i_{1}} x^{i_{3}} \cdots x^{i_{p}} \in W \otimes \Omega^{1}(W),
\end{aligned}
$$

where $\delta_{i i_{2}}$ is the Kronecker delta. It follows that for any (homogeneous) element $v \in W$,

$$
\alpha(v)= \pm \sum_{p \geqslant 2} \sum_{i_{1}, i_{3}, \ldots, i_{p}} \frac{1}{(p-2) !}\left[e_{i_{1}}, v, \ldots, e_{i_{p}}\right]_{p} d x^{i_{1}} x^{i_{3}} \cdots x^{i_{p}} \in W \otimes \Omega(W) .
$$

If $\left\{F^{r} \mathfrak{g}\right\}_{r \geq 1}$ is the lower central filtration of $\mathfrak{g}$ (see definition (4.1)), we set $F^{r} W:=F^{r} \mathfrak{g}[1]$ for $r \geq 1$. By equation (4.4), $\alpha\left(F^{j} W \otimes \Omega(W)\right) \subset F^{j+1} W \otimes \Omega(W)$ for all $j$. Hence the image of $\alpha^{r}$ is contained in $F^{r+1} W \otimes \Omega(W)$ for all $r$. Since $F^{r} W=0$ for $r \gg 0, \alpha^{r}=0$ for $r \gg 0$. This verifies that $\alpha$ is nilpotent, as desired.

By [44, Theorem 4.3] (see also [13, Theorem 5.3]), the map $\mathrm{I}_{\mathrm{HKR}}{ }^{\circ} \iota_{J(\alpha)^{\frac{1}{2}}}$ defines a quasi-isomorphism of complexes that induces an algebra isomorphism on cohomology. Since $J(\alpha)=1$, the desired proposition follows from Lemma 4.1.

Lemma 4.1. The inclusion $\mathrm{D}_{\text {poly }}^{*}(\mathcal{A}) \hookrightarrow \mathrm{C}_{\oplus}^{*}(\mathcal{A}, \mathcal{A})$ is a quasi-isomorphism of DG algebras.

Proof. The inclusion $\mathrm{D}_{\text {poly }}^{*}(\mathcal{A}) \hookrightarrow \mathrm{C}_{\oplus}^{*}(\mathcal{A}, \mathcal{A})$ is clearly compatible with cup products. It therefore suffices to check that it is a quasi-isomorphism. Let $\mathcal{A}_{0}:=(\operatorname{Sym}(V), 0)$ denote the DG algebra with trivial differential that is isomorphic to $\mathcal{A}$ as a graded algebra. First, we check that the HKR map

$$
\mathrm{I}_{\mathrm{HKR}}^{\circ}: \mathcal{A}_{0} \otimes \operatorname{Sym}(W[-1]) \rightarrow \mathrm{C}_{\oplus}^{*}\left(\mathcal{A}_{0}, \mathcal{A}_{0}\right)
$$

is a quasi-isomorphism. Indeed, $\mathrm{C}_{\oplus}^{*}\left(\mathcal{A}_{0}, \mathcal{A}_{0}\right)$ may be identified with the complex $\operatorname{Hom}\left(\mathcal{A}_{0} \otimes_{\pi} \boldsymbol{B} \mathcal{A}_{0} \otimes_{\pi}\right.$ $\mathcal{A}_{0}, \mathcal{A}_{0}$ ), where Hom is in the category of graded $\mathcal{A}_{0}$-bimodules. Here, $\mathcal{A}_{0} \otimes_{\pi} \boldsymbol{B} \mathcal{A}_{0} \otimes_{\pi} \mathcal{A}_{0}$ is viewed as the free resolution of $\mathcal{A}_{0}$ (as a graded $\mathcal{A}_{0}$-bimodule) whose term in homological degree $i$ is $\mathcal{A}_{0} \otimes \overline{\mathcal{A}}_{0} \otimes i \otimes \mathcal{A}_{0}$. On the other hand, since $\operatorname{Sym}^{c}(V[1])$ is Koszul dual to $\mathcal{A}_{0}, \mathcal{A}_{0} \otimes_{\tau} \operatorname{Sym}^{c}(V[1]) \otimes_{\tau} \mathcal{A}_{0}$ also yields a free resolution of $\mathcal{A}_{0}$ as a graded $\mathcal{A}_{0}$-bimodule, where $\tau: \operatorname{Sym}^{c}(V[1]) \rightarrow \mathcal{A}_{0}$ denotes the corresponding twisting cochain. The map $\operatorname{Sym}^{c}(V[1]) \rightarrow \boldsymbol{B} \mathcal{A}_{0}$ of coalgebras corresponding to $\tau$ induces a quasiisomorphism $\mathrm{C}_{\oplus}^{*}\left(\mathcal{A}_{0}, \mathcal{A}_{0}\right) \rightarrow \mathcal{A}_{0} \otimes \operatorname{Sym}(W[-1])$ whose inverse is the HKR map (4.5).

Now, observe that the fact that map (4.5) is a quasi-isomorphism implies that $\mathrm{I}_{\mathrm{HKR}}: \mathcal{V} \rightarrow \mathrm{C}_{\oplus}^{*}(\mathcal{A}, \mathcal{A})$ is a quasi-isomorphism. Indeed, converting cohomological grading to homological (and vice versa), we can view $\mathcal{V}$ and $\mathrm{C}_{\oplus}^{*}(\mathcal{A}, \mathcal{A})$ as direct sum complexes of the double complexes $V_{* *}$ and $C_{* *}$ with $V_{p q}=$ 
$\left(\mathcal{A} \otimes \operatorname{Sym}^{-p}(W[-1])\right)_{q}$ and $C_{p q}=\operatorname{Hom}\left(\mathcal{A}^{\otimes-p}, \mathcal{A}\right)_{q}$, respectively. Each of these double complexes is concentrated in the second and third quadrants, and I HKR defines a map of double complexes $V_{* *} \rightarrow C_{* *}$. The canonical row filtrations yield spectral sequences converging to $\mathrm{H}^{*}[\mathcal{V}]$ and $\mathrm{HH}_{\oplus}^{*}(\mathcal{A}, \mathcal{A})$, respectively. The map induced by $\mathrm{I}_{\mathrm{HKR}}$ on the $E^{1}$-page is precisely the map (4.5). By a standard comparison theorem for spectral sequences (see, e.g., [61, Theorem 5.2.12]), we conclude that $\mathrm{I}_{\mathrm{HKR}}$ is a homology isomorphism, since so is the map (4.5). ${ }^{4}$ Finally, since $\mathrm{I}_{\mathrm{HKR}}$ factors through $\mathcal{V} \rightarrow \mathrm{D}_{\text {poly }}^{*}(\mathcal{A})$, and this last map is a quasi-isomorphism, the inclusion of $\mathrm{D}_{\text {poly }}^{*}(\mathcal{A})$ in $\mathrm{C}_{\oplus}^{*}(\mathcal{A}, \mathcal{A})$ is a quasi-isomorphism as well.

Let $\mathrm{D}(\mathcal{A})$ denote the algebra of differential operators on $\mathcal{A}$ - that is, the DG subalgebra of $\operatorname{End}_{k}(\mathcal{A})$ generated by $\mathcal{A}$ and $\operatorname{Der}_{k}(\mathcal{A})$. Note that, modulo trivial modifications, the arguments of [54, Section 4] go through in the DG setting, showing that $\mathrm{D}_{\text {poly }}^{*}(\mathcal{A})$ splits into a direct sum of subcomplexes

$$
\mathrm{D}_{\text {poly }}^{*}(\mathcal{A}) \cong \bigoplus_{p=0}^{\infty} \operatorname{Sym}^{p}\left(\mathcal{L}_{\mathcal{A}}(\mathrm{D}(\mathcal{A}))\right)
$$

where $\mathcal{L}_{\mathcal{A}}(\mathrm{D}(\mathcal{A})$ ) denotes the free Lie algebra generated over $\mathcal{A}$ by $\mathrm{D}(\mathcal{A})$ (to which the Hochschild differential indeed restricts). The $\mathcal{A}$-module structure on $\mathrm{D}(\mathcal{A})$ is given by the natural left multiplication, which makes $\mathrm{D}(\mathcal{A})$ a semifree $\mathrm{DG} \mathcal{A}$-module. Setting $\mathcal{V}^{p}:=\mathcal{A} \otimes \operatorname{Sym}^{p}(W[-1])$, we see as in [54, Section 4.2] that the map $\mathrm{I}_{\mathrm{HKR}}$ restricts to a quasi-isomorphism

$$
\mathrm{I}_{\mathrm{HKR}}: \mathcal{V}^{p} \rightarrow \operatorname{Sym}^{p}\left(\mathcal{L}_{\mathcal{A}}(\mathrm{D}(\mathcal{A}))\right)
$$

for each $p$. Now, the subcomplex $\operatorname{Sym}^{p}\left(\mathcal{L}_{\mathcal{A}}(\mathrm{D}(\mathcal{A}))\right)$ of $\mathrm{D}_{\text {poly }}^{*}(\mathcal{A})$ is the image of a projection operator

$$
e^{(p), *}: \mathrm{D}_{\text {poly }}^{*}(\mathcal{A}) \rightarrow \mathrm{D}_{\text {poly }}^{*}(\mathcal{A})
$$

that is the restriction of a projection operator $e^{(p), *}$ on $\mathrm{C}_{\oplus}^{*}(\mathcal{A}, \mathcal{A})$. The latter has an explicit combinatorial definition in terms of Eulerian idempotents [46, Section 4.5]. The operators $e^{(p), *}$ define the Hodge decomposition of Hochschild cohomology:

$$
\mathrm{C}_{\oplus}^{*}(\mathcal{A}, \mathcal{A}) \cong \bigoplus_{p=0}^{\infty} \mathrm{C}_{\oplus}^{(p), *}(\mathcal{A}, \mathcal{A}), \quad \operatorname{HH}_{\oplus}^{*}(\mathcal{A}, \mathcal{A}) \cong \bigoplus_{p=0}^{\infty} \mathrm{HH}_{\oplus}^{(p), *}(\mathcal{A}, \mathcal{A}) .
$$

Lemma 4.2. The cup product on $\mathrm{HH}_{\oplus}^{*}(\mathcal{A}, \mathcal{A})$ preserves the Hodge decomposition. More precisely,

$$
\mathrm{HH}_{\oplus}^{(p), *}(\mathcal{A}, \mathcal{A}) \cup \mathrm{HH}_{\oplus}^{(q), *}(\mathcal{A}, \mathcal{A}) \subseteq \mathrm{HH}_{\oplus}^{(p+q), *}(\mathcal{A}, \mathcal{A}) .
$$

Proof. The previous argument shows that the Hochschild-Kostant-Rosenberg map intertwines the natural Hodge decomposition $\mathcal{V} \cong \oplus_{p} \mathcal{V}^{p}$ with that of $\mathrm{C}_{\oplus}^{*}(\mathcal{A}, \mathcal{A})$. By Lemma 4.1, it induces isomorphisms for each $p \geq 0$

$$
\mathrm{I}_{\mathrm{HKR}}: \mathrm{H}^{*}\left[\mathcal{V}^{p}\right] \cong \mathrm{HH}_{\oplus}^{(p), *}(\mathcal{A}, \mathcal{A}) .
$$

Since the product on $\mathcal{V}$ preserves the Hodge decomposition $\mathcal{V} \cong \oplus_{p} \mathcal{V}^{p}$, the lemma follows from Proposition 4.1.

Remark. For $d>1$, it has been shown that the cup product on the higher Hochschild cohomology $\mathrm{HH}^{S^{d}, *}(A, A)$ of a commutative DG algebra $A$ (with finite-dimensional cohomology in each degree)

${ }^{4}$ Note that this argument only uses the fact that $\mathcal{A}$ is a graded symmetric algebra with differential. That the Chevalley-Eilenberg cochain algebra of $\mathfrak{g}$ is of this form, however, uses the nilpotency of $\mathfrak{g}$. 
preserves the Hodge decomposition [29, Section 6]. This statement is, however, not true in general for $\mathrm{HH}^{S^{1}, *}(A, A)=\mathrm{HH}^{*}(A, A)$.

Proposition 4.2. Let $\mathfrak{a} \in \mathrm{DGLA}_{k}$ be as in Theorem 1.2, and let $\mathfrak{g}$ denote the minimal $L_{\infty}$-model of $\mathfrak{a}$. Then the cup product and the Gerstenhaber bracket on $\mathrm{HH}^{*}\left(\mathcal{U}_{\mathfrak{a}}, \mathcal{U}_{\mathfrak{a}}\right)$ preserve the Lie-Hodge decomposition - that is,

$$
\begin{aligned}
& \operatorname{HH}^{(p), *}(\mathfrak{a}) \cup \operatorname{HH}^{(q), *}(\mathfrak{a}) \subseteq \operatorname{HH}^{(p+q), *}(\mathfrak{a}) \\
& {\left[\operatorname{HH}^{(p), *}(\mathfrak{a}), \operatorname{HH}^{(q), *}(\mathfrak{a})\right]_{G} \subseteq \operatorname{HH}^{(p+q-1), *}(\mathfrak{a}) .}
\end{aligned}
$$

Proof. We begin by showing that there is a Hodge-decomposition-preserving isomorphism of algebras $\mathrm{HH}^{*}\left(\mathcal{U}_{\mathfrak{a}}, \mathcal{U}_{\mathfrak{a}}\right) \cong \mathrm{HH}_{\oplus}^{*}(\mathcal{A}, \mathcal{A})$, where $\mathcal{A}$ is the Chevalley-Eilenberg cochain algebra of $\mathfrak{g}$. Since $\mathcal{A}$ is neither finite dimensional (it is only locally finite dimensional) nor bigraded, we are not in a position to quote results from the literature (see, e.g., [39, Theorem 3.5] and [34]) for our purpose. Let $\mathcal{C}:=\mathcal{C}_{*}(\mathfrak{g} ; k)$ denote the Chevalley-Eilenberg chain coalgebra of $\mathfrak{g}$. Note that $\mathcal{C}=\mathcal{A}^{*}$, the graded linear dual of $\mathcal{A}$. The obvious degree -1 map $\mathcal{C}:=\mathcal{C}_{*}(\mathfrak{g} ; k) \rightarrow \mathfrak{g}$ is a generalised twisting cochain, ${ }^{5}$ which we denote by $\eta$. The unit of the adjunction $\boldsymbol{\Omega}_{\mathrm{Com}}: \mathrm{DGCC}_{k / k} \rightleftarrows$ DGLA $_{k}: \mathcal{C}_{*}$ gives a weak equivalence of DG coalgebras $\mathcal{C} \rightarrow \mathcal{C}_{*}\left(\boldsymbol{\Omega}_{\text {Com }}(\mathcal{C}) ; k\right)$ (see, e.g., [3, Section 6.2]). Let $\iota: \mathcal{C} \rightarrow \boldsymbol{\Omega}_{\mathrm{Com}}(\mathcal{C})$ denote the corresponding (canonical) twisting cochain and let $\boldsymbol{f}=\left(f_{1}, f_{2}, \ldots\right): \mathfrak{g} \rightarrow \boldsymbol{\Omega}_{\mathrm{Com}}(\mathcal{C})$ denote the corresponding $L_{\infty^{-}}$ morphism. Composition with $\boldsymbol{f}$ defines an $L_{\infty}$-morphism $\operatorname{Hom}(\mathcal{C}, \mathfrak{g}) \rightarrow \operatorname{Hom}\left(\mathcal{C}, \boldsymbol{\Omega}_{\mathrm{Com}}(\mathcal{C})\right)$, which we continue to denote by $f$. Explicitly, for $\varphi_{1}, \ldots, \varphi_{n} \in \operatorname{Hom}(\mathcal{C}, \mathfrak{g})$,

$$
f_{n}\left(\varphi_{1}, \ldots, \varphi_{n}\right)=f_{n} \circ\left(\varphi_{1} \otimes \cdots \otimes \varphi_{n}\right) \circ \Delta^{n-1},
$$

where $\Delta^{n-1}: \mathcal{C} \rightarrow \mathcal{C}^{\otimes n}$ denotes the $n$-iterated coproduct for $n \geq 2$ (with $\Delta^{0}=\mathrm{Id}$ ). Clearly, $\boldsymbol{f}(\eta)=\iota$. Next, observe that $\operatorname{Hom}(\mathcal{C}, \mathfrak{g})$ is complete with respect to the decreasing filtration $F^{n} \operatorname{Hom}(\mathcal{C}, \mathfrak{g}):=$ $\operatorname{Hom}\left(\operatorname{Sym}^{\geqslant n}(\mathfrak{g}[1]), \mathfrak{g}\right)$. Hence, by [62, Theorem 3.21] (see also [18, Proposition 1]), there is a twisted $L_{\infty}$-morphism $\boldsymbol{f}^{\eta}: \operatorname{Hom}^{\eta}(\mathcal{C}, \mathfrak{g}) \rightarrow \operatorname{Hom}^{\iota}\left(\mathcal{C}, \boldsymbol{\Omega}_{\mathrm{Com}}(\mathcal{C})\right)$, whose $n$th Taylor coefficient is given by the formula (compare [62, Definition 3.20])

$$
f_{n}^{\eta}\left(\varphi_{1}, \ldots, \varphi_{n}\right)=\sum_{k=0}^{\infty} \frac{1}{k !} f_{n+k}\left(\eta, \ldots, \eta, \varphi_{1}, \ldots, \varphi_{n}\right) .
$$

Using this, it is not difficult to verify the commutativity of the diagram

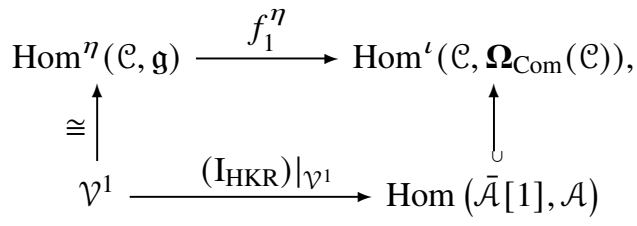

where the vertical arrow on the right is given by taking graded linear duals followed by composition by the inclusion $\overline{\mathrm{C}}[-1] \hookrightarrow \boldsymbol{\Omega}_{\mathrm{Com}}(\mathrm{C})$.

Since $\operatorname{Sym}^{p}(\mathfrak{g})$ is finite dimensional for each $p$, there is an isomorphism of DG algebras

$$
\bigoplus_{p} \operatorname{Hom}^{\eta}\left(\mathcal{C}, \operatorname{Sym}^{p}(\mathfrak{g})\right) \cong \bigoplus_{p} v^{p},
$$

${ }^{5}$ Recall that if $C$ is a (coaugmented, conilpotent) cocommutative DG coalgebra and $\mathcal{L}$ is an $L_{\infty}$-algebra, a generalised twisting cochain $\tau: C \rightarrow \mathcal{L}$ is defined to be an element of degree -1 in the convolution $L_{\infty}$-algebra Hom $(\bar{C}, \mathcal{L})$ satisfying the generalised Maurer-Cartan equation: $\partial \tau+\sum_{n \geq 2} \frac{1}{n !} m_{n}(\tau, \ldots, \tau)=0$ (see, e.g., [26, Definition 4.3]). Such generalised twisting cochains in Hom $(\bar{C}, \mathcal{L})$ are in (natural) bijection with the morphisms of coaugmented, conilpotent cocommutative DG coalgebras from $C$ to $\mathcal{C}_{*}(\mathcal{L} ; k)$. 
where the (convolution) algebra structure on the left-hand side is induced by the coproduct on $\mathcal{C}$ and the product on $\operatorname{Sym}(\mathfrak{g})$. As in Theorem 1.2, let $C$ denote a finite-dimensional cocommutative DG coalgebra Koszul dual to $\mathfrak{a}$. By [47, Theorem 10.3.15], there is an $L_{\infty}$-quasi-isomorphism $\mathfrak{a} \stackrel{\sim}{\rightarrow} \mathfrak{g}$. It follows that there are weak equivalences of DG coalgebras

$$
C \rightarrow \mathcal{C}_{*}(\mathfrak{a} ; k) \rightarrow \mathcal{C}
$$

Let $\tau$ denote the (generalised) twisting cochain $C \rightarrow \mathcal{C} \stackrel{\eta}{\rightarrow} \mathfrak{g}$. Let $\rho$ denote the (generalised) twisting cochain $C \stackrel{(4.7)}{\rightarrow} \mathcal{C} \stackrel{\iota}{\rightarrow} \boldsymbol{\Omega}(\mathcal{C})$. The commutativity of diagram (4.6) implies that there is a commutative diagram

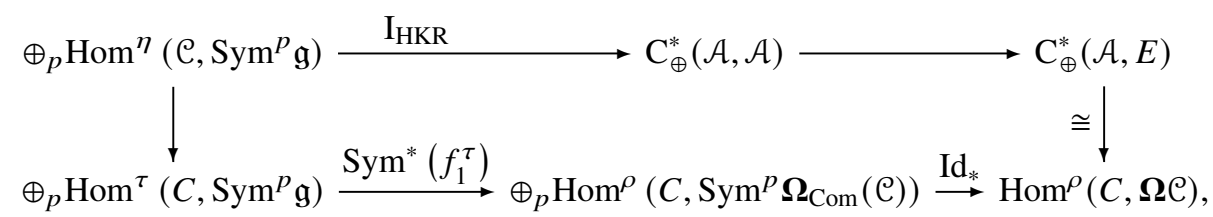

where the second arrow in the upper row is induced by the algebra homomorphism $\mathcal{A} \rightarrow E:=C^{*}$ obtained by applying graded linear duals to the map (4.7) and the last arrow on the lower row is induced by the natural symmetrisation map. Note that $\operatorname{Hom}^{\eta}\left(\mathcal{C}, \operatorname{Sym}^{p}(\mathfrak{g})\right)\left(\operatorname{resp} ., \operatorname{Hom}^{\tau}\left(C, \operatorname{Sym}^{p}(\mathfrak{g})\right)\right)$ may be identified with $\operatorname{Hom}_{\text {DG CoMode }_{\mathcal{C}}}\left(\mathcal{C}, \mathcal{C} \otimes_{\eta} \operatorname{Sym}^{p}(\mathfrak{g})\right)\left(\operatorname{resp}_{\cdot}, \operatorname{Hom}_{\text {DG CoMode }_{\mathcal{C}}}\left(C, \mathcal{C} \otimes_{\eta} \operatorname{Sym}^{p}(\mathfrak{g})\right)\right)$. Under this identification, the map $\operatorname{Hom}^{\eta}\left(\mathcal{C}, \operatorname{Sym}^{p}(\mathfrak{g})\right) \rightarrow \operatorname{Hom}^{\tau}\left(C, \operatorname{Sym}^{p}(\mathfrak{g})\right)$ is induced by the weak equivalence $C \rightarrow \mathcal{C}$ of DG $\mathcal{C}$-comodules. Since $\mathcal{C} \otimes_{\eta} \operatorname{Sym}^{p}(\mathfrak{g})$ is a fibrant (see [38, 42]) DG $\mathcal{C}$-comodule, this map is a quasi-isomorphism for each $p$ (see [35, Section 9.7]).

Now, since $C$ is conilpotent and finite dimensional, $\bar{E}^{n}=0$ for $n \gg 0$, which implies that $\operatorname{Hom}(C, \mathfrak{g})$ is complete with respect to the filtration $F^{n} \operatorname{Hom}(C, \mathfrak{g}):=\bar{E}^{\geqslant n} \otimes \mathfrak{g}$. Hence, by (minor modification of the proof of) [18, Proposition 1, Part 5], $\boldsymbol{f}$ being a quasi-isomorphism implies that $f_{1}^{\tau}$ is a quasiisomorphism. Since $\mathrm{I}_{\mathrm{HKR}}$ is a quasi-isomorphism by Lemma 4.1 and $\mathrm{Id}_{*}$ is an isomorphism of complexes, the map $\mathrm{C}_{\oplus}^{*}(\mathcal{A}, \mathcal{A}) \rightarrow \mathrm{C}_{\oplus}^{*}(\mathcal{A}, E)$ is a quasi-isomorphism of DG algebras. Thus, there is a zigzag of DG algebra maps

$$
\mathrm{C}_{\oplus}^{*}(\mathcal{A}, \mathcal{A}) \rightarrow \mathrm{C}_{\oplus}^{*}(\mathcal{A}, E) \leftarrow \mathrm{C}_{\oplus}^{*}(E, E),
$$

where the first arrow is quasi-isomorphism. We claim that the second arrow in the diagram (4.8) is also a quasi-isomorphism. Filter the complex $\mathrm{C}_{\oplus}^{*}(\mathcal{A}, E)$ by the subcomplexes $F_{q}\left(\mathrm{C}_{\oplus}^{*}(\mathcal{A}, E)\right):=$ $\oplus_{p} \operatorname{Hom}\left(\mathcal{A}^{\otimes p}, E_{\leqslant q}\right)$ and similarly for $\mathrm{C}_{\oplus}^{*}(E, E)$. Since $E$ is finite dimensional, the filtrations on these complexes are finite, and the associated spectral sequences converge. It is easy to see that $\mathrm{C}_{\oplus}^{*}(E, E) \rightarrow$ $\mathrm{C}_{\oplus}^{*}(\mathcal{A}, E)$ induces an isomorphism between the $E^{1}$-pages of these spectral sequences. Hence, by a standard comparison theorem, this map of complexes is a quasi-isomorphism.

Since the Hodge decomposition on all these Hochschild cochain complexes is combinatorially defined by the Eulerian idempotents, the induced isomorphism of algebras on cohomologies

$$
\mathrm{HH}_{\oplus}^{*}(\mathcal{A}, \mathcal{A}) \cong \mathrm{HH}_{\oplus}^{*}(E, E)
$$

preserves the Hodge decomposition as well. It follows from Corollaries 2.2 and 2.3 that there is an isomorphism of algebras preserving Hodge decomposition:

$$
\mathrm{HH}^{*}(\mathcal{U} \mathfrak{a}, \mathcal{U} \mathfrak{a}) \cong \mathrm{HH}_{\oplus}^{*}(\mathcal{A}, \mathcal{A}) .
$$

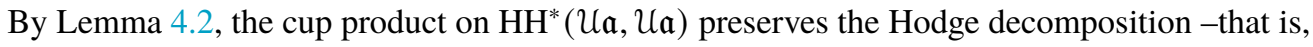

$$
\mathrm{HH}^{(p), *}(\mathfrak{a}) \cup \mathrm{HH}^{(q), *}(\mathfrak{a}) \subseteq \mathrm{HH}^{(p+q), *}(\mathfrak{a}) .
$$


Finally, define the BV operator $\Delta: \mathrm{HH}^{*}(\mathcal{U} \mathfrak{a}, \mathcal{U} \mathfrak{a}) \rightarrow \mathrm{HH}^{*-1}(\mathcal{U} \mathfrak{a}, \mathcal{U} \mathfrak{a})$ by $\Delta:=\Psi^{-1} \circ B \circ \Psi$, where $B$ denotes the Connes differential. By [7, Thm. 2.2], $B$ decreases the Lie-Hodge degree by 1 . It follows from Lemma 3.2 that $\left.\Delta\left(\mathrm{HH}^{(p), *}(\mathcal{U} \mathfrak{a}, \mathcal{U} \mathfrak{a})\right) \subseteq \mathrm{HH}^{(p-1), *}(\mathcal{U} \mathfrak{a}, \mathcal{U} \mathfrak{a})\right)$. By [31, Theorem 3.4.3],

$$
[a, b]_{G}=\Delta(a \cup b)-\Delta(a) \cup b-(-1)^{|a|} a \cup \Delta(b) .
$$

It follows from (4.9) that $[a, b]_{G} \in \operatorname{HH}^{(p+q-1), *}(\mathcal{U} \mathfrak{a}, \mathcal{U} \mathfrak{a})$ for all elements $a \in \operatorname{HH}^{(p), *}(\mathcal{U} \mathfrak{a}, \mathcal{U} \mathfrak{a})$ and $b \in \mathrm{HH}^{(q), *}\left(\mathcal{U}_{\mathfrak{a}}, \mathcal{U} \mathfrak{a}\right)$.

Proof (of Theorem 1.2). Since the minimal $L_{\infty}$-model of $\mathfrak{a}$ is finite dimensional, nonnegatively graded and nilpotent, $\mathrm{HH}^{(p), *}(\mathfrak{a}) \cup \mathrm{HH}^{(q), *}(\mathfrak{a}) \subset \mathrm{HH}^{(p+q), *}(\mathfrak{a})$ by Proposition 4.2. By [7, Theorem 2.2], $B\left(\mathrm{HC}_{*}^{(p)}(\mathfrak{a})\right) \subset \mathrm{HH}_{*}^{(p-1)}(\mathfrak{a})$ for all $p \geq 1$. Since $\mathfrak{a}$ is Koszul dual to a finite-dimensional $C \in$ $\operatorname{DGCC}_{k / k}$ equipped with a nondegenerate cyclic pairing, we have $\Psi^{-1}\left(\operatorname{HH}_{*}^{(p-1)}(\mathfrak{a})\right)=\operatorname{HH}^{(p-1), *}(\mathfrak{a})$ by Lemma 3.2. Thus,

$$
\begin{aligned}
I\left[\Psi\left[\Psi^{-1}\left(B\left(\mathrm{HC}_{*}^{(p)}(\mathfrak{a})\right)\right) \cup \Psi^{-1}\left(B\left(\mathrm{HC}_{*}^{(q)}(\mathfrak{a})\right)\right)\right]\right] & \subseteq I\left[\Psi\left[\operatorname{HH}^{(p-1), *}(\mathfrak{a}) \cup \operatorname{HH}^{(q-1), *}(\mathfrak{a})\right]\right] \\
& \subseteq I\left[\Psi\left[\mathrm{HH}^{(p+q-2), *}(\mathfrak{a})\right]\right] \\
& =I\left[\operatorname{HH}_{*}^{(p+q-2)}(\mathfrak{a})\right] \\
& \subseteq \operatorname{HC}_{*}^{(p+q-2)}(\mathfrak{a}) .
\end{aligned}
$$

The equality follows from Lemma 3.2, and the last inclusion is by [7, Theorem 2.2]. The theorem now follows immediately from Proposition 3.2.

Assume that $\mathfrak{a} \in \mathrm{DGLA}_{k}^{+}$satisfies the conditions of Theorem 1.2. Assume further that a finitedimensional $C \in \mathrm{DGCC}_{k / k}$ that is Koszul dual to a carries a nondegenerate cyclic pairing. By Theorem 3.2, there is an action of $\overline{\mathrm{HC}}_{*}(\mathcal{U} \mathfrak{a})$ on $\overline{\mathrm{HH}}_{*}(\mathcal{U} \mathfrak{a})$ making the latter a graded Lie module over the former. The following result strengthening [7, Theorem 3.4] holds under the foregoing conditions:

Theorem 4.1. For all $p, q \geqslant 1,\left\{\mathrm{HC}_{*}^{(p)}(\mathfrak{a}), \mathrm{HH}_{*}^{(q)}(\mathfrak{a})\right\} \subseteq \mathrm{HH}_{*}^{(p+q-2)}(\mathfrak{a})$.

Proof. Note that

$$
\begin{aligned}
\Psi\left[\Psi^{-1}\left(B\left(\mathrm{HC}^{(p)}(\mathfrak{a})\right)\right), \Psi^{-1}\left(\mathrm{HH}^{(q)}(\mathfrak{a})\right)\right]_{G} & \subseteq \Psi\left[\operatorname{HH}^{(p-1), *}(\mathfrak{a}), \mathrm{HH}^{(q), *}(\mathfrak{a})\right]_{G} \\
& \subseteq \Psi\left(\operatorname{HH}^{(p+q-2), *}(\mathfrak{a})\right) \\
& =\mathrm{HH}_{*}^{(p+q-2)}(\mathfrak{a}) .
\end{aligned}
$$

The first inclusion follows from [7, Theorem 2.2] and Lemma 3.2, the second from Proposition 4.2 and the equality from Lemma 3.2. The theorem follows now from Proposition 3.3.

\subsection{Application to string topology}

Let $X$ be a simply connected space of finite rational type. Recall [23] that associated to $X$ are a commutative cochain DG $\mathbb{Q}$-algebra $\mathcal{A}_{X}$, called the Sullivan model of $X$, and a connected chain DG Lie $\mathbb{Q}$-algebra $\mathfrak{a}_{X}$, called the Quillen model of $X$. The Quillen and Sullivan models determine the rational homotopy of $X$, and are Koszul dual to each other in the sense that there is a quasi-isomorphism of DG algebras

$$
\mathcal{C}^{*}\left(\mathfrak{a}_{X} ; \mathbb{Q}\right) \stackrel{\sim}{\longrightarrow} \mathcal{A}_{X},
$$

where $\mathcal{C}^{*}$ denotes the Chevalley-Eilenberg cochain complex with trivial coefficients. 
Now, let $\mathcal{L} X=\operatorname{Map}\left(S^{1}, X\right)$ denote the free loop space over $X$. In the case when $X$ is a simply connected closed oriented manifold of dimension $d$, Chas and Sullivan [14] constructed a product called the loop product - on the (reduced) rational homology of $\mathcal{L} X$ :

$$
\text { • : } \overline{\mathrm{H}}_{*}(\mathcal{L} X, \mathbb{Q}) \otimes \overline{\mathrm{H}}_{*}(\mathcal{L} X, \mathbb{Q}) \rightarrow \overline{\mathrm{H}}_{*-d}(\mathcal{L} X, \mathbb{Q}) \text {. }
$$

The free loop space $\mathcal{L} X$ is equipped with a natural circle action via rotation of loops. In addition to the usual homology, one may therefore consider the $S^{1}$-equivariant homology (resp., reduced $S^{1}$ equivariant homology) $\mathrm{H}^{S^{1}}(\mathcal{L} X, \mathbb{Q})\left(\right.$ resp., $\left.\overline{\mathrm{H}}_{*}^{S^{1}}(\mathcal{L} X, \mathbb{Q})\right)$ of $\mathcal{L} X$. The two homology theories are related by the Gysin long exact sequence

$$
\overline{\mathrm{H}}_{n-1}^{S^{1}}(\mathcal{L} X, \mathbb{Q}) \stackrel{\mathbb{M}}{\rightarrow} \overline{\mathrm{H}}_{n}(\mathcal{L} X, \mathbb{Q}) \stackrel{p_{*}}{\rightarrow} \overline{\mathrm{H}}_{n}^{S^{1}}(\mathcal{L} X, \mathbb{Q}) \stackrel{D}{\rightarrow} \overline{\mathrm{H}}_{n-2}^{S^{1}}(\mathcal{L} X, \mathbb{Q}),
$$

where $D$ stands for the Gysin map and $p: \mathcal{L} X \times E S^{1} \rightarrow \mathcal{L} X \times_{S^{1}} E S^{1}$ is the canonical projection. The string bracket on $\overline{\mathrm{H}}^{S^{1}}(\mathcal{L} X, \mathbb{Q})$ is the bilinear map

$$
\{-,-\}: \overline{\mathrm{H}}^{S^{1}}(\mathcal{L} X, \mathbb{Q}) \otimes \overline{\mathrm{H}}^{S^{1}}(\mathcal{L} X, \mathbb{Q}) \rightarrow \overline{\mathrm{H}}^{S^{1}}(\mathcal{L} X, \mathbb{Q})
$$

induced by the loop product (compare [14]):

$$
\{a, b\}=(-1)^{|a|+d} p_{*}(\mathbb{M}(a) \bullet \mathbb{M}(b)) .
$$

As mentioned in the introduction, the following theorem is a combination of well-known results ([32, Theorem V.1.1] and [23, Theorem 26.5]; see also [10, 36, 37, 58]):

Theorem 4.2. There are natural isomorphisms of graded vector spaces

$$
\alpha_{X}: \overline{\mathrm{HH}}_{*}\left(\mathcal{U} \mathfrak{a}_{X}\right) \stackrel{\sim}{\rightarrow} \overline{\mathrm{H}}_{*}(\mathcal{L} X, \mathbb{Q}), \quad \beta_{X}: \overline{\mathrm{HC}}_{*}\left(\mathcal{U a}_{X}\right) \stackrel{\sim}{\rightarrow} \overline{\mathrm{H}}_{*}^{S^{1}}(\mathcal{L} X, \mathbb{Q}),
$$

identifying the Connes periodicity sequence for $\mathcal{U a}_{X}$ with the Gysin long exact sequence for the $S^{1}$ equivariant homology of $\mathcal{L} X$.

On the other hand, the finite coverings of the circle $\varphi^{n}: S^{1} \rightarrow S^{1}, e^{i \theta} \mapsto e^{n i \theta}$, give natural maps $\varphi_{X}^{n}: \mathcal{L} X \rightarrow \mathcal{L} X$, one for each $n \geq 0$, which induce Frobenius (power) operations on homology:

$$
\Phi_{X}^{n}: \overline{\mathrm{H}}_{*}(\mathcal{L} X, \mathbb{Q}) \rightarrow \overline{\mathrm{H}}_{*}(\mathcal{L} X, \mathbb{Q}), \quad \tilde{\Phi}_{X}^{n}: \overline{\mathrm{H}}_{*}^{S^{1}}(\mathcal{L} X, \mathbb{Q}) \rightarrow \overline{\mathrm{H}}_{*}^{S^{1}}(\mathcal{L} X, \mathbb{Q})
$$

By [7, Theorem 4.1], the isomorphisms $\alpha_{X}$ and $\beta_{X}$ of Theorem 4.2 restrict to isomorphisms of (graded) vector spaces

$$
\mathrm{HH}_{*}^{(p)}\left(\mathcal{U a}_{X}\right) \stackrel{\sim}{\rightarrow} \overline{\mathrm{H}}_{*}^{(p)}(\mathcal{L} X, \mathbb{Q}), \quad \mathrm{HC}_{*}^{(p)}\left(\mathfrak{a}_{X}\right) \stackrel{\sim}{\rightarrow} \overline{\mathrm{H}}_{*}^{S^{1},(p-1)}(\mathcal{L} X, \mathbb{Q}),
$$

where the targets are common eigenspaces of the endomorphisms $\Phi_{X}^{n}$ and $\tilde{\Phi}_{X}^{n}$ with eigenvalues $n^{p}$ :

$$
\overline{\mathrm{H}}_{*}^{(p)}(\mathcal{L} X, \mathbb{Q}):=\bigcap_{n \geq 0} \operatorname{Ker}\left(\Phi_{X}^{n}-n^{p} \mathrm{Id}\right), \quad \overline{\mathrm{H}}_{*}^{S^{1},(p)}(\mathcal{L} X, \mathbb{Q}):=\bigcap_{n \geq 0} \operatorname{Ker}\left(\tilde{\Phi}_{X}^{n}-n^{p} \mathrm{Id}\right) .
$$

Thus, we have the Hodge-type decompositions

$$
\overline{\mathrm{H}}_{*}(\mathcal{L} X, \mathbb{Q})=\bigoplus_{p=0}^{\infty} \overline{\mathrm{H}}_{*}^{(p)}(\mathcal{L} X, \mathbb{Q}), \quad \overline{\mathrm{H}}_{*}^{S^{1}}(\mathcal{L} X, \mathbb{Q})=\bigoplus_{p=0}^{\infty} \overline{\mathrm{H}}_{*}^{S^{1},(p)}(\mathcal{L} X, \mathbb{Q}),
$$


and the Gysin long exact sequence (4.10) decomposes into a direct sum of exact sequences

$$
\overline{\mathrm{H}}_{n-1}^{S^{1},(p)}(\mathcal{L} X, \mathbb{Q}) \stackrel{\mathbb{M}}{\rightarrow} \overline{\mathrm{H}}_{n}^{(p)}(\mathcal{L} X, \mathbb{Q}) \stackrel{p_{*}}{\rightarrow} \overline{\mathrm{H}}_{n}^{S^{1},(p-1)}(\mathcal{L} X, \mathbb{Q}) \stackrel{D}{\rightarrow} \overline{\mathrm{H}}_{n-2}^{S^{1},(p)}(\mathcal{L} X, \mathbb{Q}) .
$$

By [7, Theorem 4.2], the string bracket gives $\overline{\mathrm{H}}_{*}^{S^{1}}(\mathcal{L} X, \mathbb{Q})$ the structure of a filtered Lie algebra with respect to the filtration

$$
F_{p} \overline{\mathrm{H}}_{*}^{S^{1}}(\mathcal{L} X, \mathbb{Q}):=\bigoplus_{q \leq p+1} \overline{\mathrm{H}}_{*}^{S^{1},(q)}(\mathcal{L} X, \mathbb{Q})
$$

The following result strengthens [24, Theorem 2] in the rationally elliptic case:

Theorem 4.3. Let $X$ be a simply connected closed oriented manifold of rationally elliptic type. Then the loop product preserves the Hodge decomposition - that is,

$$
\overline{\mathrm{H}}_{*}^{(p)}(\mathcal{L} X, \mathbb{Q}) \bullet \overline{\mathrm{H}}_{*}^{(q)}(\mathcal{L} X, \mathbb{Q}) \subseteq \overline{\mathrm{H}}_{*}^{(p+q)}(\mathcal{L} X, \mathbb{Q}) .
$$

Let $\mathfrak{a}_{X}$ be a Quillen model of $X$. Recall the isomorphism $\alpha_{X}: \operatorname{HH}_{*}\left(\mathcal{U} \mathfrak{a}_{X}\right) \cong \mathrm{H}_{*}(\mathcal{L} X, \mathbb{Q})$ of Theorem 4.2. The following proposition is a consequence of [25, Theorem D and Proposition 8]:

Proposition 4.3. The isomorphism $\alpha_{X}$ identifies the loop product on $\mathrm{H}_{*}(\mathcal{L} X, \mathbb{Q})$ with the product

$$
\mathrm{HH}_{*}\left(\mathcal{U}_{\mathfrak{a}_{X}}\right) \otimes \mathrm{HH}_{*}\left(\mathcal{U}_{\mathfrak{a}_{X}}\right) \stackrel{\bullet}{\rightarrow} \mathrm{HH}_{*}\left(\mathcal{U}_{\mathfrak{a}_{X}}\right), \quad a \otimes b \mapsto \Psi\left(\Psi^{-1}(a) \cup \Psi^{-1}(b)\right) .
$$

Proof. Define $d:=\operatorname{dim} X$. Recall that the map $\alpha_{X}^{*}$ gives a natural isomorphism $\mathrm{H}^{*}(\mathcal{L} X, \mathbb{Q}) \cong$ $\mathrm{H}^{*}\left(\mathfrak{a}_{X} ; \mathcal{U} \mathfrak{a}_{X}^{\vee}\right)$, where $\mathcal{U} \mathfrak{a}_{X}^{\vee}$ denotes the graded linear dual of $\mathfrak{a}_{X}$, both sides of which we identify. By [25, Theorem D], the isomorphism $[X] \cap-: \mathrm{H}^{d-*}\left(\mathfrak{a}_{X} ; \mathcal{U}_{X}^{\vee}\right) \rightarrow \mathrm{H}_{*}\left(\mathfrak{a}_{X} ; \mathcal{U}_{X}^{\vee}\right)$ transforms the coproduct on the right-hand side to the dual of the loop product on $\mathrm{H}^{*}\left(\mathfrak{a}_{X} ; \mathcal{U} \mathfrak{a}_{X}^{\vee}\right)$. The graded linear dual of this isomorphism therefore transforms the loop product on $\mathrm{H}_{*}(\mathcal{L} X, \mathbb{Q})$ to the product on $\mathrm{H}^{*}\left(\mathfrak{a}_{X} ; \mathfrak{U} \mathfrak{a}_{X}\right)$. Let $C$ denote the Lambrechts-Stanley model [41] of $X$. By (the proof of) Lemma 3.2, the isomorphism $[X] \cap-$ is induced by the map $\phi \otimes \operatorname{Id}_{\mathcal{U} \mathfrak{a}_{X}^{\vee}}: E \otimes \mathcal{U}_{X}^{\vee} \rightarrow C[-d] \otimes \mathcal{U} \mathfrak{a}_{X}^{\vee}$, where $E:=C^{*}$ and $\phi: E \rightarrow C[-d]$ is the isomorphism induced by the cyclic pairing on $C$. It follows that the map $\phi^{*}: E[d] \rightarrow C$ coincides with $\phi[d]$, whence the graded linear dual of the map $[X] \cap-: \mathrm{H}^{d-*}\left(\mathfrak{a}_{X} ; \mathcal{U} \mathfrak{a}_{X}^{\vee}\right) \rightarrow \mathrm{H}_{*}\left(\mathfrak{a}_{X} ; \mathcal{U} \mathfrak{a}_{X}^{\vee}\right)$ coincides with the map $[X] \cap-: \mathrm{H}^{d-*}\left(\mathfrak{a}_{X} ; \mathcal{U} \mathfrak{a}_{X}\right) \rightarrow \mathrm{H}_{*}\left(\mathfrak{a}_{X} ; \mathcal{U} \mathfrak{a}_{X}\right)$. By Lemma 3.2, this in turn is identified with the isomorphism $\Psi: \mathrm{HH}^{d-*}\left(\mathcal{U} \mathfrak{a}_{X}, \mathcal{U} \mathfrak{a}_{X}\right) \rightarrow \mathrm{HH}_{*}\left(\mathcal{U} \mathfrak{a}_{X}\right)$. Since the product on $\mathrm{H}^{*}\left(\mathfrak{a}_{X} ; \mathcal{U} \mathfrak{a}_{X}\right)$ is identified with the cup product on $\mathrm{HH}^{*}\left(\mathcal{U} \mathfrak{a}_{X}, \mathcal{U} \mathfrak{a}_{X}\right)$ by Proposition 2.2, the proposition follows.

Proof (of Theorem 4.3). Since the minimal $L_{\infty}$-model of $\mathfrak{a}_{X}$ is finite dimensional and nilpotent, Proposition 4.2 applies to $\mathfrak{a}_{X}$. By Proposition 4.2 and Lemma 3.2,

$$
\mathrm{HH}_{*}^{(p)}\left(\mathfrak{a}_{X}\right) \bullet \mathrm{HH}_{*}^{(q)}\left(\mathfrak{a}_{X}\right) \subseteq \mathrm{HH}_{*}^{(p+q)}\left(\mathfrak{a}_{X}\right) .
$$

The desired result is therefore immediate from Proposition 4.3 and [7, Theorem 4.1], by which $\alpha_{X}$ identifies $\mathrm{HH}_{*}^{(p)}\left(\mathfrak{a}_{X}\right)$ with $\mathrm{H}_{*}^{(p)}(\mathcal{L} X, \mathbb{Q})$ for all $p$.

The following lemma, which is known to experts, is an immediate consequence of Theorem 4.2 and Propositions 3.2 and 4.3:

Lemma 4.3. The isomorphism $\beta_{X}: \overline{\mathrm{HC}}_{*}\left(\mathcal{U a}_{X}\right) \stackrel{\sim}{\rightarrow} \overline{\mathrm{H}}_{*} S^{1}(\mathcal{L} X, \mathbb{Q})$ identifies the string bracket on $\overline{\mathrm{H}}_{*}^{S^{1}}(\mathcal{L} X, \mathbb{Q})$ with the derived Poisson bracket on $\overline{\mathrm{HC}}_{*}\left(\mathcal{U} \mathfrak{a}_{X}\right)$ induced by the Poincare duality pairing on its Koszul dual.

We are now in position to give a proof of our first theorem, stated in the introduction. 
Proof (of Theorem 1.1). Since the minimal $L_{\infty}$-model of $\mathfrak{a}_{X}$ is finite dimensional and nilpotent, and since the Koszul dual of $\mathfrak{a}_{X}$ equipped with the Poincaré duality pairing has a finite-dimensional model (namely, the graded linear dual of the Lambrechts-Stanley model), Theorem 1.1 follows from Lemma 4.3, Theorem 1.2 and [7, Theorem 4.1].

By Theorem 3.2, the Poincaré duality pairing on the Koszul dual of $\mathfrak{a}_{X}$ also induces an action of $\overline{\mathrm{HC}}_{*}\left(\mathcal{U} \mathfrak{a}_{X}\right)$ on $\mathrm{HH}_{*}\left(\mathcal{U} \mathfrak{a}_{X}\right)$, making the latter a graded Lie module over the former. By Theorem 4.2, we have a graded Lie action of $\overline{\mathrm{H}}_{*}^{S^{1}}(\mathcal{L} X, \mathbb{Q})$ (with string topology bracket) on $\mathrm{H}_{*}(\mathcal{L} X, \mathbb{Q})$. The following result strengthens [7, Theorem 4.3(ii)] in the case when $X$ is rationally elliptic:

Theorem 4.4. Assume that $X$ is rationally elliptic. Then

$$
\left\{\overline{\mathrm{H}}_{*}^{S^{1},(p)}(\mathcal{L} X, \mathbb{Q}), \overline{\mathrm{H}}_{*}^{(q)}(\mathcal{L} X, \mathbb{Q})\right\} \subseteq \overline{\mathrm{H}}_{*}^{(p+q-1)}(\mathcal{L} X, \mathbb{Q}) .
$$

Proof. Since the minimal $L_{\infty}$-model of $\mathfrak{a}_{X}$ is finite dimensional and nilpotent, and since the Koszul dual of $\mathfrak{a}_{X}$ equipped with the Poincaré duality pairing has a finite-dimensional model (namely, the graded linear dual of the Lambrechts-Stanley model [41]), the desired result follows immediately from Theorem 4.1 and [7, Theorem 4.1].

Acknowledgements. The first author is grateful to the Department of Mathematics of Indiana University (Bloomington) and the Forschungsinstitut für Mathematik (ETH, Zürich) for their hospitality during his stay in fall 2019. His research was partially supported by a 2019 Simons Fellowship and NSF grant DMS 1702372. The second author's research was partially supported by NSF grant DMS 1702323.

Conflict of Interest: None.

\section{References}

[1] L. Avramov and S. Halperin, 'Through the looking glass: A dictionary between rational homotopy theory and local algebra', Lecture Notes in Math. 1183 (1986), 1-27.

[2] Yu. Berest, X. Chen, F. Eshmatov and A. Ramadoss, 'Noncommutative Poisson structures, derived representation schemes and Calabi-Yau algebras', Contemp. Math. 583 (2012), 219-246.

[3] Yu. Berest, G. Felder, S. Patotski, A. C. Ramadoss and T. Willwacher, 'Representation homology, Lie algebra cohomology and the derived Harish-Chandra homomorphism', J. Eur. Math. Soc. JEMS 19(9) (2017), 2811-2893.

[4] Yu. Berest, G. Felder and A. Ramadoss, 'Derived representation schemes and noncommutative geometry', Contemp. Math. 607 (2014), 113-162.

[5] Yu. Berest, G. Khachatryan and A. Ramadoss, 'Derived representation schemes and cyclic homology', Adv. Math. 245 (2013), 625-689.

[6] Yu. Berest and A. Ramadoss, 'Stable representation homology and Koszul duality', J. Reine Angew. Math. 715 (2016), 143-187.

[7] Yu. Berest, A. Ramadoss and Y. Zhang, 'Dual Hodge decompositions and derived Poisson brackets', Selecta Math. (N.S.) 23 (2017), 2029-2070.

[8] R. Bocklandt and L. Le Bruyn, 'Necklace Lie algebras and noncommutative symplectic geometry', Math. Z. 240(1) (2002), 141-167.

[9] M. Bökstedt, W. C. Hsiang and I. Madsen, 'The cyclotomic trace and algebraic K-theory of spaces', Invent. Math. 111(3) (1993), 465-539.

[10] D. Burghelea and Z. Fiedorowicz, 'Cyclic homology and algebraic K-theory of spaces, II', Topology 25(3) (1986), 303-317.

[11] D. Burghelea, Z. Fiedorowicz and W. Gajda, 'Adams operations in Hochschild and cyclic homology of de Rham algebra and free loop spaces', K-Theory 4(3) (1991), 269-287.

[12] D. Burghelea, Z. Fiedorowicz and W. Gajda, 'Power maps and epicyclic spaces', J. Pure Appl. Algebra 96(1) (1994), $1-14$.

[13] D. Calaque and C. Rossi, Lectures on Duflo Isomorphisms in Lie Algebras and Complex Geometry, EMS Series of Lectures in Mathematics (European Mathematical Society, Zürich, Switzerland, 2011), viii+106.

[14] M. Chas and D. Sullivan, 'String topology', Preprint, 1999, arXiv:math/9911159.

[15] X. Chen, A. Eshmatov, F. Eshmatov and S. Yang, 'The derived non-commutative Poisson bracket on Koszul Calabi-Yau algebras', J. Noncommut. Geom. 11(1) (2017), 111-160. 
[16] W. Crawley-Boevey, 'Poisson structures on moduli spaces of representations', J. Algebra 325 (2011), $205-215$.

[17] L. de Thanhoffer de Völcsey and M. Van den Bergh, 'Calabi-Yau deformations and negative cyclic homology', J. Noncommut. Geom. 12(4) (2018), 1255-1291.

[18] V. A. Dolgushev, A Proof of Tsygan's Formality Conjecture for an Arbitrary Smooth Manifold, Ph.D. thesis, Massachusetts Institute of Technology (2005).

[19] V. G. Drinfeld, 'On quasitriangular quasi-Hopf algebras and on a group that is closely connected with Gal $(\overline{\mathbb{Q}} / \mathbb{Q})$, Leningrad Math. J., 2 (1991), 829-860.

[20] M. Duflo, 'Caractères des groupes et des algèbres de Lie résolubles', Ann. Sci. Éc. Norm. Supér. (4) 3 (1970), $23-74$.

[21] W. Dwyer, P. Hirschhorn, D. Kan and J. Smith, Homotopy Limit Functors on Model Categories and Homotopical Categories, Mathematical Surveys and Monographs vol. 113 (American Mathematical Society, Providence, RI, 2004).

[22] B. Feigin and B. Tsygan, 'Additive $K$-theory and crystalline cohomology', Funct. Anal. Appl. 19(2) (1985), $124-132$.

[23] Y. Felix, S. Halperin and J.-C. Thomas, Rational Homotopy Theory, Graduate Texts in Mathematics vol. 205 (SpringerVerlag, New York, 2001).

[24] Y. Felix and J.-C. Thomas, 'Rational BV algebra in string topology', Bull. Soc. Math. France 136(2) (2008), 311-327.

[25] Y. Felix, J.-C. Thomas and M. Vigué-Poirrier, 'Rational string topology', J. Eur. Math. Soc. JEMS 9 (2007), $123-156$.

[26] E. Getzler, 'Lie theory for nilpotent $L_{\infty}$-algebras', Ann. of Math. (2) 170(1) (2009), 271-301.

[27] E. Getzler and M. Kapranov, 'Cyclic operads and cyclic homology', in Geometry, Topology and Physics, Conf. Proc. Lecture Notes Geom. Topology, IV (International Press, Cambridge, MA, 1995), 167-201.

[28] G. Ginot, 'On the Hochschild and Harrison (co)homology of $C_{\infty}$-algebras and applications to string topology, in Deformation Spaces, Aspects in Mathematics, vol. E40 (Vieweg + Teubner, Wiesbaden, Germany, 2010), 1-51.

[29] G. Ginot, 'Hodge filtration and operations in higher Hochschild (co)homology and applications to higher string topology', in Algebraic Topology, Lecture Notes in Math. vol. 2194 (Springer, Cham, Switzerland, 2017), 1-104.

[30] V. Ginzburg, 'Noncommutative symplectic geometry, quiver varieties and operads', Math. Res. Lett. 8 (2001), 377-400.

[31] V. Ginzburg, 'Calabi-Yau algebras', Preprint, 2006, arXiv:math/0612139.

[32] T. Goodwillie, 'Cyclic homology, derivations, and the free loopspace', Topology 24 (1985), 187-215.

[33] A. Hamilton and A. Lazarev, 'Cohomology theories for homotopy algebras and noncommutative geometry', Algebr. Geom. Topol. 9(3) (2009), 1503-1583.

[34] E. Herscovich, 'Hochschild (co)homology and Koszul duality', Preprint, 2014, arXiv:1405.2247.

[35] P. S. Hirschhorn, Model categories and their localizations. Mathematical Surveys and Monographs 99, AMS, Providence, RI, 2003.

[36] J.D.S. Jones, 'Cyclic homology and equivariant homology', Invent. Math. 87 (1987), 403-423.

[37] J.D.S. Jones and J. McCleary, Hochschild Homology, Cyclic Homology, and the Cobar Construction, London Math. Soc. Lecture Note Ser. vol. 175 (Cambridge University Press, Cambridge, UK, 1992), 53-65.

[38] B. Keller, 'A-infinity algebras, modules and functor categories', Contemp. Math. 406 (2006), 67-93.

[39] B. Keller, 'Derived invariance of higher structures on the Hochschild complex' (2003). URL: https://webusers.imj-prg.fr/ bernhard.keller/publ/dih.pdf.

[40] M. Kontsevich, Formal Noncommutative Symplectic Geometry, The Gelfand Mathematical Seminars 1990-1992 (Birkhäuser, Boston, MA, 1993), 173-187.

[41] P. Lambrechts and D. Stanley, 'Poincaré duality and commutative differential graded algebras', Ann. Sci. Éc. Norm. Supér. (4) 41(4) (2008), 497-511.

[42] K. Lefèvre-Hasegawa, Sur les A-infini catègories, Preprint, 2003, arxiv:math/0310337.

[43] D. Leites, 'Introduction to the theory of supermanifolds', Uspekhi. Mat. Nauk 35(1) (1980), 1(211), 3-57.

[44] H.-Y. Liao, M. Stiénon and P. Xu, 'Formality theorem for differential graded manifolds', C. R. Math. Acad. Sci. Paris 356(1) (2018), 27-43.

[45] J.-L. Loday, 'Opérations sur l'homologie cyclique des algèbres commutatives', Invent. Math. 96(1) (1989), $205-230$.

[46] J.-L. Loday, Cyclic Homology, Grundlehren Math. Wiss. vol. 301, second edn (Springer-Verlag, Berlin, 1998).

[47] J.-L. Loday and B. Vallette, Algebraic Operads. Grundlehren Math. Wiss. vol. 346 (Springer, Heidelberg, 2012 ), xxiv+634.

[48] R. McCarthy, 'On operations for Hochschild homology', Comm. Algebra 21(8) (1993), 2947-2965.

[49] C. Negron, 'The cup product on Hochschild cohomology via twisting cochains and applications to Koszul rings', J. Pure Appl. Algebra 221(5) (2017), 1112-1133.

[50] M. Pevzner and C. Torossian, 'Isomorphisme de Duflo et cohomologie tangentielle', J. Geom. Phys. 51(4) (2004), 486-505.

[51] T. Pirashvili, 'On the PROP corresponding to bialgebras', Cah. Topol. Géom. Différ. Catég. 43(3) (2002), $221-239$.

[52] D. Quillen, Homotopical Algebra, Lecture Notes in Math. vol. 43 (Springer-Verlag, Berlin, 1967).

[53] D. Quillen, 'Rational homotopy theory', Ann. of Math. (2) 90 (1969), 205-295.

[54] A. C. Ramadoss, 'The big Chern classes and the Chern character'. Internat. J. Math. 19(6) (2008), 699-746.

[55] A. C. Ramadoss and Y. Zhang, 'Cyclic pairings and derived Poisson structures', New York J. Math. 25 (2019), 1-44.

[56] T. Tradler and M. Zeinalian, 'Infinity structure of Poincaré duality spaces', Algebr. Geom. Topol. 7 (2007), 233-260. With appendix by D. Sullivan. 
[57] T. Tradler and M. Zeinalian, 'Algebraic string operations', K-Theory 38(1) (2007), 59-82.

[58] M. Ungheretti, 'Free loop space and the cyclic bar construction', Bull. Lond. Math. Soc. 49(1) (2017), 95-101.

[59] M. Van den Bergh, 'Double Poisson algebras', Trans. Amer. Math. Soc. 360 (2008), 5711-5769.

[60] N. Wahl, 'Universal operations in Hochschild homology', J. Reine Angew. Math. 720 (2016), 81-127.

[61] C. Weibel, An Introduction to Homological Algebra, Cambridge Studies in Advanced Mathematics vol. 38 (Cambridge University Press, Cambridge, UK, 1994).

[62] A. Yekutieli, 'Continuous and twisted $L_{\infty}$ morphisms', J. Pure Appl. Algebra 207(3) (2006), 575-606. 